\title{
TWISTED INDEX THEORY ON GOOD ORBIFOLDS, II: FRACTIONAL QUANTUM NUMBERS
}

\author{
MATILDE MARCOLLI AND VARGHESE MATHAI
}

\begin{abstract}
This paper uses techniques in noncommutative geometry as developed by Alain Connes Co2], in order to study the twisted higher index theory of elliptic operators on orbifold covering spaces of compact good orbifolds, which are invariant under a projective action of the orbifold fundamental group, continuing our earlier work $[\mathrm{MM}]$. We also compute the range of the higher cyclic traces on $K$-theory for cocompact Fuchsian groups, which is then applied to determine the range of values of the Connes-Kubo Hall conductance in the discrete model of the quantum Hall effect on the hyperbolic plane, generalizing earlier results in [Bel+E+S], CHMM. The new phenomenon that we observe in our case is that the Connes-Kubo Hall conductance has plateaux at integral multiples of a fractional valued topological invariant, namely the orbifold Euler characteristic. Moreover the set of possible fractions has been determined, and is compared with recently available experimental data. It is plausible that this might shed some light on the mathematical mechanism responsible for fractional quantum numbers.
\end{abstract}

\section{INTRODUCTION}

This paper uses techniques in noncommutative geometry as developed by Alain Connes Co2] in order to prove a twisted higher index theorem for elliptic operators on orbifold covering spaces of compact good orbifolds, which are invariant under a projective action of the orbifold fundamental group. These higher indices are basically the evaluation of pairings of higher traces (which are cyclic cocycles arising from the orbifold fundamental group and the multiplier defining the projective action) with the index of the elliptic operator, considered as an element in the $K$-theory of some completion of the twisted group algebra of the orbifold fundamental group. This paper is the continuation of $\mathrm{MM}$ and generalizes the results there. The main purpose for studying the twisted higher index theorem on orbifolds is to highlight the fact that when the orbifold is not smooth, then the twisted higher index can be a fraction. In particular, we determine the range of the higher cyclic traces on $K$-theory for general cocompact Fuchsian groups. We adapt and generalize the discrete model of the quantum Hall effect of Bellissard and his collaborators [Bel+E+S and also CHMM, to the case of general cocompact Fuchsian groups and orbifolds, which can be viewed equivalently as the generalization to the equivariant context. The new phenomenon that we observe in our case is that the Connes-Kubo Hall conductance has plateaux at integral multiples of a fractional valued topological invariant, namely the orbifold Euler characteristic. The presence of denominators is caused by the presence of cone points singularities

1991 Mathematics Subject Classification. Primary: 58G11, 58G18 and 58G25.

Key words and phrases. Fractional quantum numbers, Quantum Hall Effect, hyperbolic space, orbifolds, $C^{*}-$ algebras, $K$-theory, cyclic cohomology, Fuchsian groups, Harper operator, Baum-Connes conjecture. 
and by the hyperbolic geometry on the complement of these cone points. The negative curvature of the hyperbolic structure replaces interaction and simulates, in our single electron model, the presence of Coulomb interactions. We also have a geometric term in the Hamiltonian (arising from the cone point singularities) which accounts partly for the effect of Coulomb interactions. This geometric model of interaction is fairly simple, hence the agreement of our fractions with the experimental values is only partial. Among the observed fractions, for instance, we can derive $5 / 3,4 / 3,4 / 5,2 / 3$, and 5/2 from genus one orbifolds, and $2 / 5,1 / 3,4 / 9,4 / 7,3 / 5,5 / 7,7 / 5$ from genus zero orbifolds, see $\S 5$. However, fractions like $3 / 7$ and $5 / 9$, seem unobtainable in this model, even including higher genus orbifolds. Their explanation probably requires a more sophisticated term describing the electron interaction. It is not unreasonable to expect that this term may also be geometric in nature, but we leave it to future studies.

There are currently several different models which describe the occurrence of fractional quantum numbers in the quantum Hall effect. Usually quantum field theoretic techniques are involved. Most notably, there is a sophisticated Chern-Simons theory model for the fractional quantum Hall effect developed by Frohlich and his collaborators, cf. [Froh]. Also within the quantum field theoretic formalism it can be noticed that possibly different models are needed in order to explain the occurrence of different sets of fractions. For example, the fraction $5 / 2$ requires by itself a separate model.

After reviewing some preliminary material in section 1, we establish in section 2 a twisted higher index theorem which adapts the proofs of the index theorems of Atiyah [At], Singer [Si], Connes and Moscovici [CM], and Gromov [Gr2], [Ma1], to the case of good orbifolds, that is, orbifolds whose orbifold universal cover is a smooth manifold. This theorem generalizes the twisted index theorem for 0 -traces of [MM] to the case of higher degree cyclic traces. The result can be summarized as follows. Let $\mathcal{R}$ be the algebra of rapidly decreasing sequences, i.e.

$$
\mathcal{R}=\left\{\left(a_{i}\right)_{i \in \mathbb{N}}: \sup _{i \in \mathbb{N}} i^{k}\left|a_{i}\right|<\infty \forall k \in \mathbb{N}\right\}
$$

Let $\Gamma$ be a discrete group and $\sigma$ be a multiplier on $\Gamma$. Let $\mathbb{C}(\Gamma, \sigma)$ denote the twisted group algebra. We denote the tensor product $\mathbb{C}(\Gamma, \sigma) \otimes \mathcal{R}$ by $\mathcal{R}(\Gamma, \sigma)$. Let $\Gamma \rightarrow \widetilde{M} \rightarrow M$ denote the universal orbifold cover of a compact good orbifold $M$, so that $\widetilde{M}$ is a smooth manifold. Suppose given a multiplier $\sigma$ on $\Gamma$ and assume that there is a projective $(\Gamma, \bar{\sigma})$-action on $L^{2}$ sections of $\Gamma$-invariant vector bundles over $\widetilde{M}$. By considering $(\Gamma, \bar{\sigma})$-invariant elliptic operators $D$ acting on $L^{2}$ sections of these bundles, we will define a $(\Gamma, \sigma)$-index element in $K$-theory

$$
\operatorname{Ind}_{\sigma}(D) \in K_{0}(\mathcal{R}(\Gamma, \sigma)) \text {. }
$$

We will compute the pairing of $\operatorname{Ind}_{\sigma}(D)$ with higher traces. More precisely, given a normalized group cocycle $c \in Z^{k}(\Gamma, \mathbb{C})$, we define a cyclic cocycle $\operatorname{tr}_{c} \in Z C^{k}(\mathbb{C}(\Gamma, \sigma))$ of dimension $k$ on the twisted group algebra $\mathbb{C}(\Gamma, \sigma)$, which extends continuously to a $k$-dimensional cyclic cocycle on $\mathcal{R}(\Gamma, \sigma)$. This induces a map on $K$-theory,

$$
\left[\operatorname{tr}_{c}\right]: K_{0}(\mathcal{R}(\Gamma, \sigma)) \rightarrow \mathbb{C} .
$$

A main theorem established in this paper is a cohomological formula for

$$
\operatorname{Ind}_{(c, \Gamma, \sigma)}(D)=\left[\operatorname{tr}_{c}\right]\left(\operatorname{Ind}_{\sigma}(D)\right) .
$$


Our method consists of applying the Connes-Moscovici local higher index theorem to a family of idempotents constructed from the heat operator on $\widetilde{M}$, all of which represent the $(\Gamma, \sigma)$-index.

Let $\Gamma$ be a Fuchsian group of signature $\left(g ; \nu_{1}, \ldots, \nu_{n}\right)$, that is, $\Gamma$ is the orbifold fundamental group of the 2 dimensional hyperbolic orbifold $\Sigma\left(g ; \nu_{1}, \ldots, \nu_{n}\right)$ of signature $\left(g ; \nu_{1}, \ldots, \nu_{n}\right)$, where $g \in \mathbb{Z}, g \geq 0$ denotes the genus and $2 \pi / \nu_{j}, \nu_{j} \in \mathbb{N}$ denotes the cone angles at the cone points of the orbifold. In [MM] we computed the $K$-theory of the twisted group $C^{*}$ algebra. Under the assumption that the Dixmier-Douady invariant of the multiplier $\sigma$ is trivial, we obtained

$$
K_{j}\left(C^{*}(\Gamma, \sigma)\right) \cong \begin{cases}\mathbb{Z}^{2-n+\sum_{j=1}^{n} \nu_{j}} & \text { if } j=0 \\ \mathbb{Z}^{2 g} & \text { if } j=1 .\end{cases}
$$

Here we use a result of [Ji], which is a twisted analogue of a result of Jollissant and which says in particular that, when $\Gamma$ is a cocompact Fuchsian group, then the natural inclusion map $j: \mathcal{R}(\Gamma, \sigma) \rightarrow C^{*}(\Gamma, \sigma)$ induces an isomorphism in $K$-theory

$$
K_{\bullet}(\mathcal{R}(\Gamma, \sigma)) \cong K_{\bullet}\left(C^{*}(\Gamma, \sigma)\right)
$$

Using this, together with our twisted higher index theorem for good orbifolds and some results in [MM], and under the same assumptions as before, we determine, in section 3 , the range of the higher trace on $K$-theory

$$
\left[\operatorname{tr}_{c}\right]\left(K_{0}\left(C^{*}(\Gamma, \sigma)\right)\right)=\phi \mathbb{Z}
$$

where $-\phi=2(1-g)+(\nu-n) \in \mathbb{Q}$ is the orbifold Euler characteristic of $\Sigma\left(g ; \nu_{1}, \ldots, \nu_{n}\right)$. Here we have $\quad \nu=\sum_{j=1}^{n} 1 / \nu_{j}$ and $c$ is the area 2 -cocycle on $\Gamma$, i.e. $c$ is the restriction to $\Gamma$ of the area 2-cocycle on $\operatorname{PSL}(2, \mathbb{R})$.

In section 4 we study the hyperbolic Connes-Kubo formula for the Hall conductance in the discrete model of the Quantum Hall Effect on the hyperbolic plane, where we consider Cayley graphs of Fuchsian groups which may have torsion subgroups. This generalizes the results in CHMM where only torsion-free Fuchsian groups were considered. We recall that the results in CHMM generalized to hyperbolic space the noncommutative geometry approach to the Euclidean quantum Hall effect that was pioneered by Bellissard and collaborators Bel+E+S|, Connes Co and Xia Xia]. We first relate the hyperbolic Connes-Kubo Hall conductance cyclic 2-cocycle and the area cyclic 2-cocycle on the algebra $\mathcal{R}(\Gamma, \sigma)$, and show that they define the same class in cyclic cohomology. Then we use our theorem on the range of the higher trace on $K$-theory to determine the range of values of the Connes-Kubo Hall conductance cocycle in the Quantum Hall Effect. The new phenomenon that we observe in this case is that the Hall conductance has plateaux at all energy levels belonging to any gap in the spectrum of the Hamiltonian (known as the generalized Harper operator), where it is now shown to be equal to an integral multiple of a fractional valued topological invariant $\phi$, which is the negative of the orbifold Euler characteristic of the good orbifold $\Sigma\left(g ; \nu_{1}, \ldots, \nu_{n}\right)$. If we fix the genus, then the set of possible denominators is finite by the Hurwitz theorem [Sc], and has been explicitly determined in the low genus cases [Bro]. This provides a topological explanation of the appearance of fractional quantum numbers. In the last section we compare our results with some observed values.

In section 5, we provide lists of specific examples of good 2-dimensional orbifolds for which $\phi$ is not an integer. First we observe how the presence of both the hyperbolic structure and the cone 
points is essential in order to have fractional quantum numbers. In fact, $\phi$ is an integer whenever the hyperbolic orbifold is smooth, i.e. whenever $1=\nu_{1}=\ldots=\nu_{n}$, which is the case considered in CHMM. Similarly, by direct inspection, it is possible to see that all Euclidean orbifolds also produce only integer values of $\phi$. We use the class of orbifolds which are spheres or tori with cone points, having a (singular) hyperbolic structure, to represent in our physical model some of the fractions observed in the FQHE. We also list the examples arising from quotients of low genus surfaces [Bro], and we discuss some phenomenology on the role of the orbifold points and of the minimal genus $g^{\prime}$ of the covering surface.

Summarizing, one key advantage of our model is that the fractions we get are obtained from an equivariant index theorem and are thus topological in nature. Consequently, as pointed out in Bel+E+S], the Hall conductance is seen to be stable under small deformations of the Hamiltonian. Thus, this model can be easily generalized to systems with disorder as in CHM. This is a necessary step in order to establish the presence of plateaux $B e \mathrm{H}+\mathrm{E}+\mathrm{S}$. The main limitation of our model is that there is a small number of experimental fractions that we do not obtain in our model, and we also derive other fractions which do not seem to correspond to experimentally observed values. To our knowledge, however, this is also a limitation occuring in the other models available in the literature.

Acknowledgments: We thank J. Bellissard for his encouragement and for some useful comments. The second author thanks A. Carey and K. Hannabuss for some helpful comments concerning the section 4. The first author is partially supported by NSF grant DMS-9802480. Research by the second author is supported by the Australian Research Council.

\section{Preliminaries}

Recall that, if $\mathbb{H}$ denotes the hyperbolic plane and $\Gamma$ is a Fuchsian group of signature $\left(g ; \nu_{1}, \ldots, \nu_{n}\right)$, that is, $\Gamma$ is a discrete cocompact subgroup of $P S L(2, \mathbb{R})$ of genus $g$ and with $n$ elliptic elements of order $\nu_{1}, \ldots, \nu_{n}$ respectively, then the corresponding compact oriented hyperbolic 2-orbifold of signature $\left(g ; \nu_{1}, \ldots, \nu_{n}\right)$ is defined as the quotient space

$$
\Sigma\left(g ; \nu_{1}, \ldots, \nu_{n}\right)=\Gamma \backslash \mathbb{H},
$$

where $g$ denotes the genus and $2 \pi / \nu_{j}, \nu_{j} \in \mathbb{N}$ denotes the cone angles at the cone points of the orbifold. A compact oriented 2-dimensional Euclidean orbifold is obtained in a similar manner, but with $\mathbb{H}$ replaced by $\mathbb{R}^{2}$.

All Euclidean and hyperbolic 2-dimensional orbifolds $\Sigma\left(g ; \nu_{1}, \ldots, \nu_{n}\right)$ are good, being in fact orbifold covered by a smooth surface $\Sigma_{g^{\prime}}$ cf. [Sd], i.e. there is a finite group $G$ acting on $\Sigma_{g^{\prime}}$ with quotient $\Sigma\left(g ; \nu_{1}, \ldots, \nu_{n}\right)$, where $g^{\prime}=1+\frac{\#(G)}{2}(2(g-1)+(n-\nu))$ and where $\nu=\sum_{j=1}^{n} 1 / \nu_{j}$.

For fundamental material on orbifolds, see [Sd], FuSt] and [Bro]. See also [MM], section 1.

Let $M$ be a good, compact orbifold, and $\mathcal{E} \rightarrow M$ be an orbifold vector bundle over $M$, and $\widetilde{\mathcal{E}} \rightarrow \widetilde{M}$ be its lift to the universal orbifold covering space $\Gamma \rightarrow \widetilde{M} \rightarrow M$, which is by assumption a simply-connected smooth manifold. We have a $(\Gamma, \bar{\sigma})$-action (where $\sigma$ is a multiplier on $\Gamma$ and $\bar{\sigma}$ denotes its complex conjugate) on $L^{2}(\widetilde{M})$, where we choose $\omega=d \eta$ an exact 2-form on $\widetilde{M}$ such that 
$\omega$ is also $\Gamma$-invariant, although $\eta$ is not assumed to be $\Gamma$-invariant, and the Hermitian connection

$$
\nabla=d+i \eta
$$

on the trivial line bundle over $\widetilde{M}$, with curvature $\nabla^{2}=i \omega$. The projective action is defined as follows:

Firstly, observe that since $\widetilde{\omega}$ is $\Gamma$-invariant, $0=\gamma^{*} \widetilde{\omega}-\widetilde{\omega}=d\left(\gamma^{*} \eta-\eta\right) \forall \gamma \in \Gamma$. So $\gamma^{*} \eta-\eta$ is a closed 1 -form on the simply connected manifold $\widetilde{M}$, therefore

$$
\gamma^{*} \eta-\eta=d \phi_{\gamma} \quad \forall \gamma \in \Gamma
$$

where $\phi_{\gamma}$ is a smooth function on $\widetilde{M}$ satisfying in addition,

- $\phi_{\gamma}(x)+\phi_{\gamma^{\prime}}(\gamma x)-\phi_{\gamma^{\prime} \gamma}(x)$ is independent of $x \in \widetilde{M} \forall \gamma, \gamma^{\prime} \in \Gamma$;

- $\phi_{\gamma}\left(x_{0}\right)=0$ for some $x_{0} \in \widetilde{M} \forall \in \Gamma$.

Then $\bar{\sigma}\left(\gamma, \gamma^{\prime}\right)=\exp \left(i \phi_{\gamma}\left(\gamma^{\prime} \cdot x_{0}\right)\right)$ defines a multiplier on $\Gamma$ i.e. $\bar{\sigma}: \Gamma \times \Gamma \rightarrow U(1)$ satisfies the following identity for all $\gamma, \gamma^{\prime}, \gamma^{\prime \prime} \in \Gamma$

$$
\bar{\sigma}\left(\gamma, \gamma^{\prime}\right) \bar{\sigma}\left(\gamma, \gamma^{\prime} \gamma^{\prime \prime}\right)=\bar{\sigma}\left(\gamma \gamma^{\prime}, \gamma^{\prime \prime}\right) \bar{\sigma}\left(\gamma^{\prime}, \gamma^{\prime \prime}\right)
$$

For $u \in L^{2}(\widetilde{M}, \widetilde{\mathcal{E}})$, let $S_{\gamma} u=e^{i \phi_{\gamma}} u$ and $U_{\gamma} u=\gamma^{*} u$ and $T_{\gamma}=U_{\gamma} \circ S_{\gamma}$ be the composition. Then $T$ defines a projective $(\Gamma, \bar{\sigma})$-action on $L^{2}$-spinors, i.e.

$$
T_{\gamma} T_{\gamma^{\prime}}=\bar{\sigma}\left(\gamma, \gamma^{\prime}\right) T_{\gamma \gamma^{\prime}}
$$

This defines a $(\Gamma, \bar{\sigma})$-action, provided that the Dixmier-Douady invariant $\delta(\sigma)=0$, see [MM].

As in [MM], we shall consider the twisted group von Neumann algebra $W^{*}(\Gamma, \sigma)$, the commutant of the left $\bar{\sigma}$-regular representation on $\ell^{2}(\Gamma)$ and $W^{*}(\sigma)$ as the commutant of the $(\Gamma, \bar{\sigma})$-action on $L^{2}\left(\widetilde{M}, \widetilde{\mathcal{S}^{ \pm} \otimes E}\right)$.

We have an identification (see [MM])

$$
W^{*}(\sigma) \cong W^{*}(\Gamma, \sigma) \otimes B\left(L^{2}\left(\mathcal{F},\left.\widetilde{\mathcal{E}}\right|_{\mathcal{F}}\right)\right)
$$

where $B\left(L^{2}\left(\mathcal{F},\left.\widetilde{\mathcal{E}}\right|_{\mathcal{F}}\right)\right)$ denotes the algebra of all bounded operators on the Hilbert space $L^{2}\left(\mathcal{F},\left.\widetilde{\mathcal{E}}\right|_{\mathcal{F}}\right)$, and $\mathcal{F}$ is a relatively compact fundamental domain in $\widetilde{M}$ for the action of $\Gamma$. We have a semifinite trace

$$
\operatorname{tr}: W^{*}(\sigma) \rightarrow \mathbb{C}
$$

defined as in the untwisted case due to Atiyah At,

$$
Q \rightarrow \int_{M} \operatorname{tr}\left(k_{Q}(x, x)\right) d x
$$

where $k_{Q}$ denotes the Schwartz kernel of $Q$. Note that this trace is finite whenever $k_{Q}$ is continuous in a neighborhood of the diagonal in $\widetilde{M} \times \widetilde{M}$. 
We also consider, as in $\mathrm{MM}$, the subalgebra $C^{*}(\sigma)$ of $W^{*}(\sigma)$, whose elements have the additional property of some off-diagonal decay, and one also has the identification (cf. MM]

$$
C^{*}(\sigma) \cong C^{*}(\Gamma, \sigma) \otimes \mathcal{K}\left(L^{2}\left(\mathcal{F},\left.\widetilde{\mathcal{E}}\right|_{\mathcal{F}}\right)\right)
$$

In [MM] we considered the $C^{*}$ algebra

$$
C^{*}(M)=C(P) \rtimes \mathrm{SO}(m),
$$

where $P$ is the bundle of oriented frames on the orbifold tangent bundle. The relevent K-theory is the orbifold $K$-theory

$$
K_{\text {orb }}^{0}(M) \equiv K_{0}\left(C^{*}(M)\right)=K_{0}(C(P) \rtimes \mathrm{SO}(m)) \cong K_{\mathrm{SO}(m)}^{0}(P) .
$$

In the case when $M$ is a good orbifold, one can show that the $C^{*}$ algebras $C^{*}(M)$ and $C_{0}(X) \rtimes G$ are strongly Morita equivalent, where $X$ is smooth and $G \rightarrow X \rightarrow M$ is an orbifold cover. In particular,

$$
K_{\text {orb }}^{0}(M) \cong K^{0}\left(C_{0}(X) \rtimes G\right)=K_{G}^{0}(X) .
$$

The relevant cohomology is the orbifold cohomology $H_{\text {orb }}^{j}(M)=H^{j}(X, G)$, for $j=0,1$, which is the delocalized equivariant cohomology for a finite group action on a smooth manifold [BC]. The Baum-Connes equivariant Chern character is a homomorphism

$$
\operatorname{ch}_{G}: K_{G}^{0}(X) \rightarrow H^{0}(X, G) .
$$

Let $\underline{B} \Gamma=\Gamma \backslash \underline{E} \Gamma$ be the classifying space of proper actions, as defined in [BCH]. In our case, the orbifold $\Sigma\left(g ; \nu_{1}, \ldots, \nu_{n}\right)$, viewed as the quotient space $\Gamma \backslash \mathbb{H}$, is $\underline{B} \Gamma\left(g ; \nu_{1}, \ldots, \nu_{n}\right)$. Equivalently, $\underline{B} \Gamma\left(g ; \nu_{1}, \ldots, \nu_{n}\right)$ can be viewed as the classifying space of the orbifold fundamental group $\Gamma\left(g ; \nu_{1}, \ldots, \nu_{n}\right)$.

Let $S \Gamma$ denote the set of all elements of $\Gamma$ which are of finite order. Then $S \Gamma$ is not empty, since $1 \in S \Gamma$. $\Gamma$ acts on $S \Gamma$ by conjugation, and let $F \Gamma$ denote the associated permutation module over $\mathbb{C}$, i.e.

$$
F \Gamma=\left\{\sum_{\alpha \in S \Gamma} \lambda_{\alpha}[\alpha] \mid \lambda_{\alpha} \in \mathbb{C} \quad \text { and } \quad \lambda_{\alpha}=0 \quad \text { except for a finite number of } \alpha\right\}
$$

Let $C^{k}(\Gamma, F \Gamma)$ denote the space of all antisymmetric $F \Gamma$-valued $\Gamma$-maps on $\Gamma^{k+1}$, where $\Gamma$ acts on $\Gamma^{k+1}$ via the diagonal action. The coboundary map is

$$
\partial c\left(g_{0}, \ldots, g_{k+1}\right)=\sum_{i=0}^{k+1}(-1)^{i} c\left(g_{0}, \ldots, \hat{g}_{i} \ldots g_{k+1}\right)
$$

for all $c \in C^{k}(\Gamma, F \Gamma)$ and where $\hat{g}_{i}$ means that $g_{i}$ is omitted. The cohomology of this complex is the group cohomology of $\Gamma$ with coefficients in $F \Gamma, H^{k}(\Gamma, F \Gamma)$, cf. [BCH. They also show that $H^{k}(\Gamma, F \Gamma) \cong H^{j}(\Gamma, \mathbb{C}) \oplus_{m} H^{k}\left(Z\left(C_{m}\right), \mathbb{C}\right)$, where $S \Gamma=\left\{1, C_{m} \mid m=1, \ldots\right\}$ and the isomorphism is canonical. 
Also, for any Borel measurable $\Gamma-\operatorname{map} \mu: \underline{E} \Gamma \rightarrow \Gamma$, there is an induced map on cochains

$$
\mu^{*}: C^{k}(\Gamma, F \Gamma) \rightarrow C^{k}(\underline{E} \Gamma, \Gamma)
$$

which induces an isomorphism on cohomology, $\quad \mu^{*}: H^{k}(\Gamma, F \Gamma) \cong H^{k}(\underline{E} \Gamma, \Gamma) \quad[\overline{\mathrm{BCH}}]$. Here $H^{j}(\underline{E} \Gamma, \Gamma)$ denotes the $\mathbb{Z}$-graded (delocalised) equivariant cohomology of $\underline{E} \Gamma$, which is a refinement of what was discussed earlier, and which is defined in BCH using sheaves (and cosheaves), but we will not recall the definition here.

Let $M$ be a good orbifold with orbifold fundamental group $\Gamma$. We have seen that the universal orbifold cover $\widetilde{M}$ is classified by a continuous map $f: M \rightarrow \underline{B} \Gamma$, or equivalently by a $\Gamma$-map $f: \widetilde{M} \rightarrow \underline{E} \Gamma$. The induced map is $f^{*}: H_{\text {orb }}^{j}(\underline{B} \Gamma, \mathbb{C}) \equiv H^{k}(\underline{E} \Gamma, \Gamma) \rightarrow H^{k}(\widetilde{M}, \Gamma) \equiv H_{\text {orb }}^{k}(M, \mathbb{C})$ and therefore in particular one has $f^{*}([c]) \in H_{\text {orb }}^{k}(M, \mathbb{C})$ for all $[c] \in H^{k}(\Gamma, \mathbb{C})$. This can be expressed on the level of cochains by easily modifying the procedure in $\mathrm{CM}$, and we refer to [CM] for further details.

Finally, we add here a brief comment on the assumption used throughout [MM] on the vanishing of the Dixmier-Douady invariant of the multiplier $\sigma$. We show here that the condition is indeed necessary, since we can always find examples where $\delta(\sigma) \neq 0$. Let $\Gamma$ be the Fuchsian group of signature $\left(g ; \nu_{1}, \ldots, \nu_{n}\right)$, as before. Consider the long exact sequence of the change of coefficient groups, as in MM,

$$
\begin{gathered}
\cdots H^{1}(\Gamma, U(1)) \stackrel{\delta}{\rightarrow} H^{2}(\Gamma, \mathbb{Z}) \stackrel{i_{*}}{\rightarrow} H^{2}(\Gamma, \mathbb{R}) \stackrel{e_{*}^{2 \pi \sqrt{-1}} \rightarrow}{\rightarrow} \\
H^{2}(\Gamma, U(1)) \stackrel{\delta}{\rightarrow} H^{3}(\Gamma, \mathbb{Z}) \rightarrow H^{3}(\Gamma, \mathbb{R}) .
\end{gathered}
$$

The argument of $\overline{\mathrm{MM}}$ shows that $H^{3}(\Gamma, \mathbb{R})=0$ and $H^{2}(\Gamma, \mathbb{R})=\mathbb{R}$. Moreover, we observe that $H^{1}(\Gamma, \mathbb{Z})=\operatorname{Hom}(\Gamma, \mathbb{Z}) \cong \mathbb{Z}^{2 g}, \quad H^{1}(\Gamma, \mathbb{R})=\operatorname{Hom}(\Gamma, \mathbb{R}) \cong \mathbb{R}^{2 g}$ and $H^{1}(\Gamma, U(1))=$ $\operatorname{Hom}(\Gamma, U(1)) \cong U(1)^{2 g} \times_{j=1}^{n} \mathbb{Z}_{\nu_{j}}$. Now $H^{2}(\Gamma, \mathbb{Z})=\mathbb{Z} \oplus_{j} \mathbb{Z}_{\nu_{j}}$, see Patt, which is consistent with the result in [MM] that the group of the orbifold line bundles over the orbifold $\Gamma \backslash \mathbb{H}$ has $1-n+\sum_{j=1}^{n} \nu_{j}$ generators. It is also proved in Patt that $H^{2}(\Gamma, U(1))=U(1) \times_{j=1}^{n} \mathbb{Z}_{\nu_{j}}$. Using the long exact sequence and the remarks above, we see that $H^{3}(\Gamma, \mathbb{Z})=\operatorname{Tor}\left(H^{2}(\Gamma, U(1))\right)=\times_{j=1}^{n} \mathbb{Z}_{\nu_{j}}$. Thus, in the sequence we have $\operatorname{Ker}\left(i_{*}\right)=\oplus_{j} \mathbb{Z}_{\nu_{j}}, \operatorname{Im}\left(i_{*}\right)=\mathbb{Z}=\operatorname{Ker}\left(e_{*}^{2 \pi \sqrt{-1}}\right), \operatorname{Im}\left(e_{*}^{2 \pi \sqrt{-1}}\right)=$ $U(1)$. So we can identify all the classes of multipliers with trivial Dixmier-Douady invariant with $U(1)=\operatorname{Ker}(\delta)$. Finally, we have

$$
\operatorname{Im}(\delta)=H^{3}(\Gamma, \mathbb{Z})=H^{2}(\Gamma, U(1)) / \operatorname{Ker}(\delta)=\oplus_{j} \mathbb{Z}_{\nu_{j}} .
$$

The calculations of the cohomology of the Fuchsian group $\Gamma=\Gamma\left(g ; \nu_{1}, \ldots, \nu_{n}\right)$ are summarized in the following table.

\begin{tabular}{|c|c|c|c|}
\hline$j$ & $H^{j}(\Gamma, \mathbb{Z})$ & $H^{j}(\Gamma, \mathbb{R})$ & $H^{j}(\Gamma, \mathbf{U}(1))$ \\
\hline \hline 0 & $\mathbb{Z}$ & $\mathbb{R}$ & $\mathbf{U}(1)$ \\
\hline 1 & $\mathbb{Z}^{2 g}$ & $\mathbb{R}^{2 g}$ & $\mathbf{U}(1)^{2 g} \oplus_{j} \mathbb{Z}_{\nu_{j}}$ \\
\hline 2 & $\mathbb{Z} \oplus_{j} \mathbb{Z}_{\nu_{j}}$ & $\mathbb{R}$ & $\mathbf{U}(1) \oplus_{j} \mathbb{Z}_{\nu_{j}}$ \\
\hline 3 & $\oplus_{j} \mathbb{Z}_{\nu_{j}}$ & 0 & \\
\hline
\end{tabular}




\section{TWISTED HIGHER INDEX THEOREM}

In this section, we will define the higher twisted index of an elliptic operator on a good orbifold, and establish a cohomological formula for any cyclic trace arising from a group cocycle, and which is applied to the twisted higher index. We adapt the strategy and proof in [CM] to our context.

2.1. Construction of the parametrix and the index map. Let $M$ be a compact, good orbifold, that is, the universal cover $\Gamma \rightarrow \widetilde{M} \rightarrow M$ is a smooth manifold and we will assume, as before, that there is a $(\Gamma, \bar{\sigma})$-action on $L^{2}(\widetilde{M})$ given by $T_{\gamma}=U_{\gamma} \circ S_{\gamma} \forall \gamma \in \Gamma$. Let $\widetilde{\mathcal{E}}, \widetilde{\mathcal{F}}$ be Hermitian vector bundles on $M$ and let $\widetilde{\mathcal{E}}, \widetilde{\mathcal{F}}$ be the corresponding lifts to $\Gamma$-invariants Hermitian vector bundles on $\widetilde{M}$. Then there are induced $(\Gamma, \bar{\sigma})$-actions on $L^{2}(\widetilde{M}, \widetilde{\mathcal{E}})$ and $L^{2}(\widetilde{M}, \widetilde{\mathcal{F}})$ which are also given by $T_{\gamma}=U_{\gamma} \circ S_{\gamma} \forall \gamma \in \Gamma$.

Now let $D: L^{2}(\widetilde{M}, \widetilde{\mathcal{E}}) \rightarrow L^{2}(\widetilde{M}, \widetilde{\mathcal{F}})$ be a first order $(\Gamma, \bar{\sigma})$-invariant elliptic operator. Let $U \subset \widetilde{M}$ be an open subset that contains the closure of a fundamental domain for the $\Gamma$-action on $\widetilde{M}$. Let $\psi \in C_{c}^{\infty}(\widetilde{M})$ be a compactly supported smooth function such that $\operatorname{supp}(\psi) \subset U$, and

$$
\sum_{\gamma \in \Gamma} \gamma^{*} \psi=1
$$

Let $\phi \in C_{c}^{\infty}(\widetilde{M})$ be a compactly supported smooth function such that $\phi=1$ on $\operatorname{supp}(\psi)$.

Since $D$ is elliptic, we can construct a parametrix $J$ for it on the open set $U$ by standard methods,

$$
J D u=u-H u \quad \forall u \in C_{c}^{\infty}\left(U,\left.\widetilde{\mathcal{E}}\right|_{U}\right)
$$

where $H$ has a smooth Schwartz kernel. Define the pseudodifferential operator $Q$ as

$$
Q=\sum_{\gamma \in \Gamma} T_{\gamma} \phi J \psi T_{\gamma}^{*}
$$

We compute,

$$
Q D w=\sum_{\gamma \in \Gamma} T_{\gamma} \phi J \psi D T_{\gamma}^{*} w \quad \forall w \in C_{c}^{\infty}(\widetilde{M}, \widetilde{\mathcal{E}}),
$$

since $T_{\gamma} D=D T_{\gamma} \quad \forall \gamma \in \Gamma$. Since $D$ is a first order operator, one has

$$
D(\psi w)=\psi D w+(D \psi) w
$$

so that (3) becomes

$$
=\sum_{\gamma \in \Gamma} T_{\gamma} \phi J D \psi T_{\gamma}^{*} w-\sum_{\gamma \in \Gamma} T_{\gamma} \phi J(D \psi) T_{\gamma}^{*} w .
$$

Using (2), the expression above becomes

$$
=\sum_{\gamma \in \Gamma} T_{\gamma} \psi T_{\gamma}^{*} w-\sum_{\gamma \in \Gamma} T_{\gamma} \phi H \psi T_{\gamma}^{*} w-\sum_{\gamma \in \Gamma} T_{\gamma} \phi J(D \psi) T_{\gamma}^{*} w .
$$

Therefore (3) becomes

$$
Q D=I-R_{0}
$$


where

$$
R_{0}=\sum_{\gamma \in \Gamma} T_{\gamma}(\phi H \psi+J(D \psi)) T_{\gamma}^{*}
$$

has a smooth Schwartz kernel. It is clear from the definition that one has $T_{\gamma} Q=Q T_{\gamma}$ and $T_{\gamma} R_{0}=R_{0} T_{\gamma} \quad \forall \gamma \in \Gamma$. Define

$$
R_{1}={ }^{t} R_{0}+D R_{0}^{t} Q-D Q\left({ }^{t} R_{0}\right) .
$$

Then $T_{\gamma} R_{1}=R_{1} T_{\gamma} \quad \forall \gamma \in \Gamma, R_{1}$ has a smooth Schwartz kernel and satisfies

$$
D Q=I-R_{1} .
$$

Summarizing, we have obtained the following

Proposition 2.1. Let $M$ be a compact, good orbifold and $\Gamma \rightarrow \widetilde{M} \rightarrow M$ be the universal orbifold covering space. Let $\mathcal{E}, \mathcal{F}$ be Hermitian vector bundles on $M$ and let $\widetilde{\mathcal{E}}, \widetilde{\mathcal{F}}$ be the corresponding lifts to $\Gamma$-invariants Hermitian vector bundles on $\widetilde{M}$. We will assume as before that there is a $(\Gamma, \bar{\sigma})$-action on $L^{2}(\widetilde{M})$ given by $T_{\gamma}=U_{\gamma} \circ S_{\gamma} \forall \gamma \in \Gamma$, and induced $(\Gamma, \bar{\sigma})$-actions on $L^{2}(\widetilde{M}, \widetilde{\mathcal{E}})$ and $L^{2}(\widetilde{M}, \widetilde{\mathcal{F}})$ which are also given by $T_{\gamma}=U_{\gamma} \circ S_{\gamma} \forall \gamma \in \Gamma$.

Now let $D: L^{2}(\widetilde{M}, \widetilde{\mathcal{E}}) \rightarrow L^{2}(\widetilde{M}, \widetilde{\mathcal{F}})$ be a first order $(\Gamma, \bar{\sigma})$-invariant elliptic operator. Then there is an almost local, $(\Gamma, \bar{\sigma})$-invariant elliptic pseudodifferential operator $Q$ and $(\Gamma, \bar{\sigma})$-invariant smoothing operators $R_{0}, R_{1}$ which satisfy

$$
Q D=I-R_{0} \quad \text { and } \quad D Q=I-R_{1} .
$$

Define the idempotent

$$
e(D)=\left(\begin{array}{cc}
R_{0}^{2} & \left(R_{0}+R_{0}^{2}\right) Q \\
R_{1} D & 1-R_{1}^{2}
\end{array}\right)
$$

Then $e(D) \in M_{2}(\mathcal{R}(\Gamma, \sigma))$, where $\mathcal{R}(\Gamma, \sigma)=\mathbb{C}(\Gamma, \sigma) \otimes \mathcal{R}$ is as defined in $\S 1$.

The $\mathcal{R}(\Gamma, \sigma)$-index is by fiat

$$
\left.\operatorname{Ind}_{\sigma}(D)=[e(D)]-\left[E_{0}\right] \in K_{0}(\mathcal{R}(\Gamma, \sigma))\right)
$$

where $E_{0}$ is the idempotent

$$
E_{0}=\left(\begin{array}{ll}
0 & 0 \\
0 & 1
\end{array}\right)
$$

It is not difficult to see that $\operatorname{Ind}_{\sigma}(D)$ is independent of the choice of $(\Gamma, \bar{\sigma})$-invariant parametrix $Q$ that is needed in its definition.

Let $j: \mathcal{R}(\Gamma, \sigma) \rightarrow C_{r}^{*}(\Gamma, \sigma)$ be the canonical inclusion, which induces the morphism in $K$-theory

$$
j_{*}: K_{\bullet}(\mathcal{R}(\Gamma, \sigma)) \rightarrow K_{\bullet}\left(C_{r}^{*}(\Gamma, \sigma)\right) .
$$

Then we have,

Definition. The $C_{r}^{*}(\Gamma, \sigma)$-index of a $(\Gamma, \bar{\sigma})$-invariant elliptic operator $D: L^{2}(\widetilde{M}, \widetilde{\mathcal{E}}) \rightarrow L^{2}(\widetilde{M}, \widetilde{\mathcal{F}})$ is defined as

$$
\operatorname{Ind}_{(\Gamma, \sigma)}(D)=j_{*}\left(\operatorname{Ind}_{\sigma}(D)\right) \in K_{0}\left(C_{r}^{*}(\Gamma, \sigma)\right)
$$


2.2. Heat kernels and the index map. Given $D$ as before, for $t>0$, we use the standard off-diagonal estimates for the heat kernel. Recall that the heat kernels $e^{-t D^{*} D}$ and $e^{-t D D^{*}}$ are elements in the $\mathcal{R}(\Gamma, \sigma)$ (see the appendix). Define the idempotent $e_{t}(D) \in M_{2}(\mathcal{R}(\Gamma, \sigma)$ ) (see the appendix) as follows

$$
e_{t}(D)=\left(\begin{array}{cc}
e^{-t D^{*} D} & e^{-t / 2 D^{*} D} \frac{\left(1-e^{-t D^{*} D}\right)}{D^{*} D} D^{*} \\
e^{-t / 2 D D^{*} D} & 1-e^{-t D D^{*}}
\end{array}\right) .
$$

It is sometimes known as the Wasserman idempotent.

The relationship with the idempotent $e(D)$ constructed earlier can be explained as follows. Define for $t>0$,

$$
Q_{t}=\frac{\left(1-e^{-t / 2 D^{*} D}\right)}{D^{*} D} D^{*}
$$

Then one easily verifies that $Q_{t} D=1-e^{-t / 2 D^{*} D}=1-R_{0}(t)$ and $D Q_{t}=1-e^{-t / 2 D D^{*}}=1-R_{1}(t)$. That is, $Q_{t}$ is a parametrix for $D$ for all $t>0$. Therefore one can write

$$
e_{t}(D)=\left(\begin{array}{cc}
R_{0}(t)^{2} & \left(R_{0}(t)+R_{0}(t)^{2}\right) Q_{t} \\
R_{1}(t) D & 1-R_{1}(t)^{2}
\end{array}\right)
$$

In particular, one has for $t>0$

$$
\operatorname{Ind}_{\sigma}(D)=\left[e_{t}(D)\right]-\left[E_{0}\right] \in K_{0}(\mathcal{R}(\Gamma, \sigma)) .
$$

We use the same notation as in $\mathrm{MM}$. A first order elliptic differential operator $D$ on $M$,

$$
D: L^{2}(M, \mathcal{E}) \rightarrow L^{2}(M, \mathcal{F})
$$

is by fiat a $\Gamma$-equivariant first order elliptic differential operator $\widetilde{D}$ on the smooth manifold $\widetilde{M}$,

$$
\widetilde{D}: L^{2}(\widetilde{M}, \widetilde{\mathcal{E}}) \rightarrow L^{2}(\widetilde{M}, \widetilde{\mathcal{F}}) .
$$

Given any connection $\nabla^{\widetilde{W}}$ on $\widetilde{W}$ which is compatible with the $\Gamma$ action and the Hermitian metric, we define an extension of the elliptic operator $\widetilde{D}$, to act on sections of $\widetilde{\mathcal{E}} \otimes \widetilde{W}, \widetilde{\mathcal{F}} \otimes \widetilde{W}$,

$$
\widetilde{D} \otimes \nabla^{\widetilde{W}}: \Gamma(M, \widetilde{\mathcal{E}} \otimes \widetilde{W}) \rightarrow \Gamma(M, \widetilde{\mathcal{F}} \otimes \widetilde{W})
$$

as in $\mathrm{MM}$.

2.3. Group cocycles and cyclic cocycles. Using the pairing theory of cyclic cohomology and $K$-theory, due to [Co], we will pair the $(\Gamma, \sigma)$-index of a $(\Gamma, \bar{\sigma})$-invariant elliptic operator $D$ on $\widetilde{M}$ with certain cyclic cocycles on $\mathcal{R}(\Gamma, \sigma)$. The cyclic cocycles that we consider come from normalised group cocycles on $\Gamma$. More precisely, given a normalized group cocycle $c \in Z^{k}(\Gamma, \mathbb{C})$, for $k=0, \ldots, \operatorname{dim} M$, we define a cyclic cocycle $\operatorname{tr}_{c}$ of dimension $k$ on the twisted group ring $\mathbb{C}(\Gamma, \sigma)$, which is given by

$$
\operatorname{tr}_{c}\left(a_{0} \delta_{g_{0}}, \ldots, a_{k} \delta_{g_{k}}\right)=\left\{\begin{array}{l}
a_{0} \ldots a_{k} c\left(g_{1}, \ldots, g_{k}\right) \operatorname{tr}\left(\delta_{g_{0}} \delta_{g_{1}} \ldots \delta_{g_{k}}\right) \text { if } g_{0} \ldots g_{k}=1 \\
0 \quad \text { otherwise. }
\end{array}\right.
$$


where $a_{j} \in \mathbb{C}$ for $j=0,1, \ldots, k$. To see that this is a cyclic cocycle on $\mathbb{C}(\Gamma, \sigma)$, we first define, as done in [Ji], the twisted differential graded algebra $\Omega^{\bullet}(\Gamma, \sigma)$ as the differential graded algebra of finite linear combinations of symbols

$$
g_{0} d g_{1} \ldots d g_{n} \quad g_{i} \in \Gamma
$$

with module structure and differential given by

$$
\begin{aligned}
\left(g_{0} d g_{1} \ldots d g_{n}\right) g & =\sum_{j=1}^{n}(-1)^{n-1} \sigma\left(g_{j}, g_{j+1}\right) g_{0} d g_{1} \ldots d\left(g_{j} g_{j+1}\right) \ldots d g_{n} d g \\
& +(-1)^{n} \sigma\left(g_{n}, g\right) g_{0} d g_{1} \ldots d\left(g_{n} g\right) \\
d\left(g_{0} d g_{1} \ldots d g_{n}\right) & =d g_{0} d g_{1} \ldots d g_{n}
\end{aligned}
$$

We now recall normalised group cocycles. A group $k$-cocycle is a map $h: \Gamma^{k+1} \rightarrow \mathbb{C}$ satisfying the identities

$$
\begin{aligned}
h\left(g g_{0}, \ldots g g_{k}\right) & =h\left(g_{0}, \ldots g_{k}\right) \\
0 & =\sum_{i=0}^{k+1}(-1)^{i} h\left(g_{0}, \ldots, g_{i-1}, g_{i+1} \ldots, g_{k+1}\right)
\end{aligned}
$$

Then a normalised group $k$-cocycle $c$ that is associated to such an $h$ is given by

$$
c\left(g_{1}, \ldots, g_{k}\right)=h\left(1, g_{1}, g_{1} g_{2}, \ldots, g_{1} \ldots g_{k}\right)
$$

and it is defined to be zero if either $g_{i}=1$ or if $g_{1} \ldots g_{k}=1$. Any normalised group cocycle $c \in Z^{k}(\Gamma, \mathbb{C})$ determines a $k$-dimensional cycle via the following closed graded trace on $\Omega^{\bullet}(\Gamma, \sigma)$

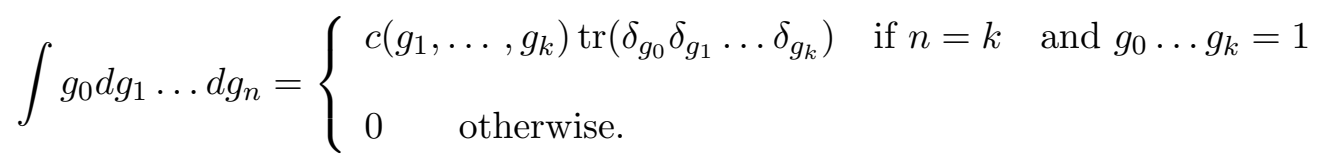

Of particular interest is the case when $k=2$, when the formula above reduces to

$$
\int g_{0} d g_{1} d g_{2}= \begin{cases}c\left(g_{1}, g_{2}\right) \sigma\left(g_{1}, g_{2}\right) & \text { if } g_{0} g_{1} g_{2}=1 \\ 0 & \text { otherwise }\end{cases}
$$

The higher cyclic trace $\operatorname{tr}_{c}$ is by fiat this closed graded trace.

2.4. Twisted higher index theorem- the cyclic cohomology version. Let $M$ be a compact orbifold of dimension $n=4 \ell$. Let $\Gamma \rightarrow \widetilde{M} \stackrel{p}{\rightarrow} M$ be the universal cover of $M$ and the orbifold fundamental group is $\Gamma$. Let $D$ be an elliptic first order operator on $M$ and $\widetilde{D}$ be the lift of $D$ to $\widetilde{M}$

$$
\widetilde{D}: L^{2}(\widetilde{M}, \widetilde{\mathcal{E}}) \rightarrow L^{2}(\widetilde{M}, \widetilde{\mathcal{F}}) .
$$

Note that $\widetilde{D}$ commutes with the $\Gamma$-action on $\widetilde{M}$. 
Now let $\omega$ be a closed 2 -form on $M$ such that $\widetilde{\omega}=p^{*} \omega=d \eta$ is exact on $\widetilde{M}$. Define $\nabla=$ $d+i \eta$. Then $\nabla$ is a Hermitian connection on the trivial line bundle over $\widetilde{M}$, and the curvature of $\nabla,(\nabla)^{2}=i \widetilde{\omega}$. Then $\nabla$ defines a projective action of $\Gamma$ on $L^{2}$ spinors on $\widetilde{M}$ as in section 1 .

Consider the twisted elliptic operator on $\widetilde{M}$,

$$
\widetilde{D} \otimes \nabla: L^{2}(\widetilde{M}, \widetilde{\mathcal{E}}) \rightarrow L^{2}(\widetilde{M}, \widetilde{\mathcal{F}})
$$

Then $\widetilde{D} \otimes \nabla$ no longer commutes with $\Gamma$, but it does commute with the projective $(\Gamma, \bar{\sigma})$ action. In $\S 2.1$, we have defined the higher index of such an operator,

$$
\operatorname{Ind}_{\sigma}(\widetilde{D} \otimes \nabla) \in K_{0}(\mathcal{R}(\Gamma, \sigma)) .
$$

Given a group cocycle $c \in Z^{2 q}(\Gamma)$, one can define the associated cyclic cocycle $\tau_{c}$ on $\mathcal{R}(\Gamma, \sigma)$ as in $\S 2.3$. Then $\tau_{c}$ induces a homomorphism on $K$-theory

$$
\left[\tau_{c}\right]: K_{0}(\mathcal{R}(\Gamma, \sigma)) \rightarrow \mathbb{R} .
$$

The real valued higher index is the image of the higher index under this homomorphism, i.e.

$$
\operatorname{Ind}_{(c, \Gamma, \sigma)}(\widetilde{D} \otimes \nabla)=\left[\tau_{c}\right]\left(\operatorname{Ind}_{\sigma}(\widetilde{D} \otimes \nabla)\right)
$$

To introduce the next theorem, we will briefly review some material on characteristic classes for orbifold vector bundles. Let $M$ be a good orbifold, that is the universal orbifold cover $\Gamma \rightarrow \widetilde{M} \rightarrow$ $M$ of $M$ is a smooth manifold. Then the orbifold tangent bundle $T M$ of $M$ can be viewed as the $\Gamma$-equivariant bundle $T \widetilde{M}$ on $\widetilde{M}$. Similar comments apply to the orbifold cotangent bundle $T^{*} M$ and, more generally, to any orbifold vector bundle on $M$. It is then clear that, choosing $\Gamma$-invariant connections on the $\Gamma$-invariant vector bundles on $\widetilde{M}$, one can define the Chern-Weil representatives of the characteristic classes of the $\Gamma$-invariant vector bundles on $\widetilde{M}$. These characteristic classes are $\Gamma$-invariant and so define cohomology classes on $M$. For further details, see [Kaw].

Theorem 2.2. Let $M$ be a compact, even dimensional, good orbifold, and let $\Gamma$ be its orbifold fundamental group. Let $\widetilde{D}$ be a first order, $\Gamma$-invariant elliptic differential operator acting on $L^{2}$ sections of $\Gamma$-invariant vector bundles on $\widetilde{M}$, where $\Gamma \rightarrow \widetilde{M} \rightarrow M$ is the universal orbifold cover of $M$. Then, for any group cocycle $c \in Z^{2 q}(\Gamma)$, one has

$$
\operatorname{Ind}_{(c, \Gamma, \sigma)}(\widetilde{D} \otimes \nabla)=\frac{q !}{(2 \pi i)^{q}(2 q !)}\left\langle T d(M) \cup \operatorname{ch}(\operatorname{symb}(D)) \cup f^{*}\left(\phi_{c}\right) \cup e^{\omega},\left[T^{*} M\right]\right\rangle
$$

where $\operatorname{Td}(M)$ denotes the Todd characteristic class of the complexified orbifold tangent bundle of $M$ which is pulled back to the orbifold cotangent bundle $T^{*} M$, ch(symb $\left.(D)\right)$ is the Chern character of the symbol of the operator $D, \phi_{c}$ is the Alexander-Spanier cocycle on $\underline{B} \Gamma$ that corresponds to the group cocycle $c$ and $f: M \rightarrow \underline{B} \Gamma$ is the map that classifies the orbifold universal cover $\widetilde{M} \rightarrow M$, cf. section 1.

Proof. Choose a bounded, almost everywhere smooth Borel cross-section $\beta: M \rightarrow \widetilde{M}$, which can then be used to define the Alexander-Spanier cocycle $\phi_{c}$ corresponding to $c \in Z^{2 q}(\Gamma)$, and such that $\left[\phi_{c}\right]=f^{*}[c] \in H^{2 q}(M)$. As in $\S 2.2$, for $t>0$, there is an index idempotent,

$$
e_{t}(D)=\left(\begin{array}{cc}
R_{0}(t)^{2} & \left(R_{0}(t)+R_{0}(t)^{2}\right) Q_{t} \\
R_{1}(t) D & 1-R_{1}(t)^{2}
\end{array}\right) \in M_{2}(\mathcal{R}(\Gamma, \sigma)),
$$


where for $t>0$,

$$
Q_{t}=\frac{\left(1-e^{-t / 2 D^{*} D}\right)}{D^{*} D} D^{*} \quad, R_{0}(t)=e^{-t / 2 D^{*} D}, \quad R_{1}(t)=e^{-t / 2 D D^{*}} .
$$

Then as in $\S 2.2$, one sees that $R_{0}(t), R_{1}(t)$ are smoothing operators and $Q_{t}$ is a parametrix for $D$ for all $t>0$. The $\mathcal{R}(\Gamma, \sigma)$-index map is then

$$
\operatorname{Ind}_{\sigma}(\widetilde{D} \otimes \nabla)=\left[e_{t}(\widetilde{D} \otimes \nabla)\right]-\left[E_{0}\right] \in K_{0}(\mathcal{R}(\Gamma, \sigma)) .
$$

where $E_{0}$ is the idempotent

$$
E_{0}=\left(\begin{array}{ll}
0 & 0 \\
0 & 1
\end{array}\right)
$$

Let $R_{t}=e_{t}(\widetilde{D} \otimes \nabla)-E_{0}$. We adapt the strategy and proof in CM to our situation.

$$
\begin{aligned}
\operatorname{Ind}_{(c, \Gamma, \sigma)}(\widetilde{D} \otimes \nabla)= & \operatorname{tr}_{c}\left(R_{t}, R_{t}, \ldots R_{t}\right) \\
= & \int_{M^{2 q+1}} \sum_{\gamma_{1}, \ldots \gamma_{2 q} \in \Gamma} c\left(1, \gamma_{1}, \ldots, \gamma_{2 q}\right) \times \\
& \operatorname{tr}\left(R_{t}\left(\beta\left(x_{0}\right), \gamma_{1} \beta\left(x_{1}\right)\right) \ldots R_{t}\left(\gamma_{2 q} \beta\left(x_{2 q}\right), \beta\left(x_{0}\right)\right)\right) d x_{0} \ldots d x_{2 q}
\end{aligned}
$$

where $\beta: M \rightarrow \widetilde{M}$ denotes a bounded measurable section. Notice that the phase term $\operatorname{tr}\left(\delta_{\gamma_{1}} \ldots \delta_{\gamma_{2 q}}\right)$ appearing in the expression for the cocycle $\operatorname{tr}_{c}$ cf. $\S 2.3$, is exactly cancelled by the twisted product of the integral kernels $R_{t}$, cf. [CHMM $\S 3$. Notice also that the right hand side of equation (5) is independent of the choice of the section $\beta$, since upon changing $\beta$ to $\gamma \cdot \beta$, we obtain

$$
\begin{aligned}
R_{t}\left(\gamma \beta\left(x_{0}\right), \gamma_{1} \gamma \beta\left(x_{1}\right)\right) \ldots R_{t}\left(\gamma_{2 q} \gamma \beta\left(x_{2 q}\right), \gamma \beta\left(x_{0}\right)\right)= & e^{i \psi_{\gamma}\left(\beta\left(x_{0}\right)\right)} R_{t}\left(\beta\left(x_{0}\right), \gamma^{-1} \gamma_{1} \gamma \beta\left(x_{1}\right)\right) e^{-i \psi_{\gamma}\left(\gamma^{-1} \gamma_{1} \gamma \beta\left(x_{1}\right)\right)} \ldots \\
& e^{i \psi_{\gamma}\left(\gamma^{-1} \gamma_{2 q} \gamma \beta\left(x_{2 q}\right)\right)} R_{t}\left(\gamma^{-1} \gamma_{2 q} \gamma \beta\left(x_{2 q}\right), \beta\left(x_{0}\right)\right) e^{-i \psi_{\gamma}\left(\beta\left(x_{0}\right)\right)} \\
= & R_{t}\left(\beta\left(x_{0}\right), \tilde{\gamma}_{1} \beta\left(x_{1}\right)\right) \ldots R_{t}\left(\tilde{\gamma}_{2 q} \beta\left(x_{2 q}\right), \beta\left(x_{0}\right)\right),
\end{aligned}
$$

where $\tilde{\gamma}_{i}=\gamma^{-1} \gamma_{i} \gamma$ which is exactly as in the case when the multiplier is trivial.

Observe that if $\beta_{U}: U \rightarrow \widetilde{M}$ is a smooth local section, then there is a unique element $\left(\gamma_{1}, \ldots, \gamma_{2 q}\right) \in \Gamma^{2 q}$ such that $\left(\beta\left(x_{0}\right), \gamma_{1} \beta\left(x_{1}\right), \ldots \gamma_{2 q} \beta\left(x_{2 q}\right)\right) \in \beta_{U}(U)^{2 q+1}$. Moreover, we have the equality $c\left(1, \gamma_{1}, \ldots, \gamma_{2 q}\right)=\phi_{c}\left(x_{0}, x_{1}, \ldots x_{2 q}\right) \quad$ (and $\phi_{c}=0$ otherwise), where $\phi_{c}$ denotes the $\Gamma$-equivariant (Alexander-Spanier) $2 q$-cocycle on $\widetilde{M}$ representing the pullback $f^{*}(c)$ of the group 2 -cocycle via the classifying map $f$. Since $R_{t}$ is mainly supported near the diagonal as $t \rightarrow 0$, and using the equivariance of $R_{t}$, one sees that

$$
\operatorname{Ind}_{(c, \Gamma, \sigma)}(\widetilde{D} \otimes \nabla)=\lim _{t \rightarrow 0} \int_{M^{2 q+1}} \phi_{c}\left(x_{0}, x_{1}, \ldots x_{2 q}\right) \operatorname{tr}\left(R_{t}\left(x_{0}, x_{1}\right) \ldots R_{t}\left(x_{2 q}, x_{0}\right)\right) d x_{0} d x_{1} \ldots d x_{2 q}
$$

where we have identified $M$ with a fundamental domain for the $\Gamma$ action on $\widetilde{M}$. The proof is completed by applying the local higher index Theorems 3.7 and 3.9 in [CM, to obtain the desired cohomological formula (4) for $\operatorname{Ind}_{(c, \Gamma, \sigma)}(\widetilde{D} \otimes \nabla)$. 


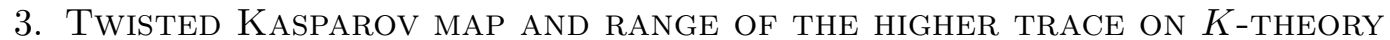

In this section, we compute the range of the $2-\operatorname{trace} \operatorname{tr}_{c}$ on $K$-theory of the twisted group $C^{*}$ algebra, where $c$ is a 2-cocycle on the group, generalising the work of [CHMM. Suppose as before that $\Gamma$ is a discrete, cocompact subgroup of $P S L(2, \mathbb{R})$ of signature $\left(g ; \nu_{1}, \ldots, \nu_{n}\right)$. That is, $\Gamma$ is the orbifold fundamental group of a compact hyperbolic orbifold $\Sigma\left(g ; \nu_{1}, \ldots, \nu_{n}\right)$ of signature $\left(g ; \nu_{1}, \ldots, \nu_{n}\right)$. Then for any multiplier $\sigma$ on $\Gamma$ such that $\delta(\sigma)=0$, one has the twisted Kasparov isomorphism,

$$
\mu_{\sigma}: K_{\text {orb }}^{\bullet}\left(\Sigma\left(g ; \nu_{1}, \ldots, \nu_{n}\right)\right) \rightarrow K_{\bullet}\left(C_{r}^{*}(\Gamma, \sigma)\right),
$$

Proposition 2.14 in [MM]. Its construction is recalled in this section, as we need to refine it by factoring it through the $K$-theory of the dense subalgebra $\mathcal{R}(\Gamma, \sigma)$ of $C_{r}^{*}(\Gamma, \sigma)$. This is necessary in order to be able to use the pairing theory of Connes [CD], [CM] between higher cyclic traces and $K$-theory. We note that using a result of [Ji], that $\mathcal{R}(\Gamma, \sigma)$ is indeed a dense subalgebra of $C_{r}^{*}(\Gamma, \sigma)$ in our case. In particular, given any projection $P$ in $C_{r}^{*}(\Gamma, \sigma)$ there is both a projection $\tilde{P}$ in the same $K_{0}$ class but lying in the dense subalgebra $\mathcal{R}(\Gamma, \sigma)$. This fact will also be utilized in the next section. On the other hand, by the results of the current section, given any such projection $P$ there is a higher topological index that we can associate to it cf. Theorem 3.3. The main result we prove here is that the range of the 2 -trace $\operatorname{tr}_{c}$ on $K$-theory of the twisted group $C^{*}$ algebra is always an integer multiple of a rational number. This will enable us to compute the range of values of the Hall conductance in the quantum Hall effect on hyperbolic space, generalizing the results in $\mathrm{CHMM}$.

3.1. Twisted Kasparov map. Let $\Gamma$ be as before, that is, $\Gamma$ is the orbifold fundamental group of the hyperbolic orbifold $\Sigma\left(g ; \nu_{1}, \ldots, \nu_{n}\right)$. Then for any multiplier $\sigma$ on $\Gamma$, we will factor the twisted Kasparov isomorphism,

$$
\mu_{\sigma}: K_{\text {orb }}^{\bullet}\left(\Sigma\left(g ; \nu_{1}, \ldots, \nu_{n}\right)\right) \rightarrow K_{\bullet}\left(C_{r}^{*}(\Gamma, \sigma)\right)
$$

in [MM through the $K$-theory of the dense subalgebra $\mathcal{R}(\Gamma, \sigma)$ of $C_{r}^{*}(\Gamma, \sigma)$.

Let $\mathcal{E} \rightarrow \Sigma\left(g ; \nu_{1}, \ldots, \nu_{n}\right)$ be an orbifold vector bundle over $\Sigma\left(g ; \nu_{1}, \ldots, \nu_{n}\right)$ defining an element $[\mathcal{E}]$ in $K^{0}\left(\Sigma\left(g ; \nu_{1}, \ldots, \nu_{n}\right)\right)$. As in [Kaw, one can form the twisted Dirac operator $\not_{\mathcal{E}}^{+}$: $L^{2}\left(\Sigma\left(g ; \nu_{1}, \ldots, \nu_{n}\right), \mathcal{S}^{+} \otimes \mathcal{E}\right) \rightarrow L^{2}\left(\Sigma\left(g ; \nu_{1}, \ldots, \nu_{n}\right), \mathcal{S}^{-} \otimes \mathcal{E}\right)$ where $\mathcal{S}^{ \pm}$denote the $\frac{1}{2}$ spinor bundles over $\Sigma\left(g ; \nu_{1}, \ldots, \nu_{n}\right)$. One can lift the twisted Dirac operator $\not_{\mathcal{E}}^{+}$as above, to a $\Gamma$-invariant operator $\widetilde{\partial_{\mathcal{E}}^{+}}$on $\mathbb{H}=\widetilde{\Sigma}\left(g ; \nu_{1}, \ldots, \nu_{n}\right)$, which is the universal orbifold cover of $\Sigma\left(g ; \nu_{1}, \ldots, \nu_{n}\right)$,

$$
\widetilde{\partial_{\mathcal{E}}^{+}}: L^{2}(\mathbb{H}, \widetilde{\mathcal{S}+\otimes \mathcal{E}}) \rightarrow L^{2}\left(\mathbb{H}, \widetilde{\mathcal{S}^{-} \otimes \mathcal{E}}\right)
$$

For any multiplier $\sigma$ of $\Gamma$ with $\delta([\sigma])=0$, there is a $\mathbb{R}$-valued 2-cocycle $\zeta$ on $\Gamma$ with $[\zeta] \in$ $H^{2}(\Gamma, \mathbb{R})$ such that $\left[e^{2 \pi \sqrt{-1} \zeta}\right]=[\sigma]$. By the argument of [MM], section 2.2, we know that we have an isomorphism $H^{2}(\Gamma, \mathbb{R}) \cong H^{2}\left(\Gamma_{g^{\prime}}, \mathbb{R}\right)$, and therefore there is a 2-form $\omega$ on $\Sigma_{g^{\prime}}$ such that $\left[e^{2 \pi \sqrt{-1} \omega}\right]=[\sigma]$. Of course, the choice of $\omega$ is not unique, but this will not affect the results that we are concerned with. Let $\widetilde{\omega}$ denote the lift of $\omega$ to the universal cover $\mathbb{H}$. Since the hyperbolic plane $\mathbb{H}$ is contractible, it follows that $\widetilde{\omega}=d \eta$ where $\eta$ is a 1 -form on $\mathbb{H}$ which is not in general $\Gamma$ invariant. Now $\nabla=d+i \eta$ is a Hermitian connection on the trivial complex line bundle on $\mathbb{H}$. 
Note that the curvature of $\nabla$ is $\nabla^{2}=i \tilde{\omega}$. Consider now the twisted Dirac operator $\tilde{\partial}_{\mathcal{E}}^{+}$which is twisted again by the connection $\nabla$,

$$
\widetilde{\partial_{\mathcal{E}}^{+}} \otimes \nabla: L^{2}\left(\mathbb{H}, \widetilde{\mathcal{S}^{+} \otimes \mathcal{E}}\right) \rightarrow L^{2}\left(\mathbb{H}, \widetilde{\mathcal{S}^{-} \otimes \mathcal{E}}\right) .
$$

It does not commute with the $\Gamma$ action, but it does commute with the projective $(\Gamma, \bar{\sigma})$-action which is defined by the connection $\nabla$ as in $\S 1$. In section 2.1 , we have defined the higher index of such an operator

$$
\operatorname{Ind}_{\sigma}\left(\widetilde{\partial_{\mathcal{E}}^{+}} \otimes \nabla\right) \in K_{0}(\mathcal{R}(\Gamma, \sigma)),
$$

where as before, $\mathcal{R}$ denotes the algebra of rapidly decreasing sequences on $\mathbb{Z}^{2}$.

Then the twisted Kasparov map (6) is

$$
\mu_{\sigma}([\mathcal{E}])=j_{*}\left(\operatorname{Ind}_{\sigma}\left(\widetilde{\partial_{\mathcal{E}}^{+}} \otimes \nabla\right)\right)=\operatorname{Ind}_{(\Gamma, \sigma)}\left(\widetilde{\partial_{\mathcal{E}}^{+}} \otimes \nabla\right) \in K_{0}\left(C^{*}(\Gamma, \sigma)\right),
$$

where $j: \mathcal{R}(\Gamma, \sigma)=\mathbb{C}(\Gamma, \sigma) \otimes \mathcal{R} \rightarrow C_{r}^{*}(\Gamma, \sigma) \otimes \mathcal{K}$ is the natural inclusion map, and as before, $\mathcal{K}$ denotes the algebra of compact operators. Then

$$
j_{*}: K_{0}(\mathcal{R}(\Gamma, \sigma)) \rightarrow K_{0}\left(C_{r}^{*}(\Gamma, \sigma)\right)
$$

is the induced map on $K_{0}$. The twisted Kasparov map was defined for certain torsionfree groups in [CHMM] and the general case in [Ma1]. It is related to the Baum-Connes assembly map [BC], BCH, as is discussed in Ma1.

3.2. Range of the higher trace on $K$-theory. The first step in the proof is to show that given a bounded group cocycle $c \in Z^{2}(\Gamma)$ we may define canonical pairings with $K^{0}\left(\Sigma\left(g ; \nu_{1}, \ldots, \nu_{n}\right)\right)$ and $K_{0}\left(C_{r}^{*}(\Gamma, \sigma)\right)$ which are related by the twisted Kasparov isomorphism, by adapting some of the results of Connes and Connes-Moscovici to the twisted case. As $\Sigma\left(g ; \nu_{1}, \ldots, \nu_{n}\right)=\underline{B} \Gamma$ is a negatively curved orbifold, we know (by [Mos] and [Gr]) that degree 2 cohomology classes in $H^{2}(\Gamma)$ have bounded representatives i.e. bounded 2-cocycles on $\Gamma$. The bounded group 2-cocycle $c$ may be regarded as a skew symmetrised function on $\Gamma \times \Gamma \times \Gamma$, so that we can use the results in section 2 to obtain a cyclic 2 -cocycle $\operatorname{tr}_{c}$ on $\mathbb{C}(\Gamma, \sigma) \otimes \mathcal{R}$ by defining:

$$
\operatorname{tr}_{c}\left(f^{0} \otimes r^{0}, f^{1} \otimes r^{1}, f^{2} \otimes r^{2}\right)=\operatorname{Tr}\left(r^{0} r^{1} r^{2}\right) \sum_{g_{0} g_{1} g_{2}=1} f^{0}\left(g_{0}\right) f^{1}\left(g_{1}\right) f^{2}\left(g_{2}\right) c\left(1, g_{1}, g_{1} g_{2}\right) \sigma\left(g_{1}, g_{2}\right) .
$$

Since the only difference with the expression obtained in $\mathrm{CM}$ is $\sigma\left(g_{1}, g_{2}\right)$, and since $\left|\sigma\left(g_{1}, g_{2}\right)\right|=1$, we can use Lemma 6.4, part (ii) in CM and the assumption that $c$ is bounded, to obtain the necessary estimates which show that in fact $\operatorname{tr}_{c}$ extends continuously to the bigger algebra $\mathcal{R}(\Gamma, \sigma)$. By the pairing of cyclic theory and $K$-theory in $\mathrm{CD}$, one obtains an additive map

$$
\left[\operatorname{tr}_{c}\right]: K_{0}(\mathcal{R}(\Gamma, \sigma)) \rightarrow \mathbb{R}
$$

Explicitly, $\left[\operatorname{tr}_{c}\right]([e]-[f])=\widetilde{\operatorname{tr}}_{c}(e, \cdots, e)-\widetilde{\operatorname{tr}}_{c}(f, \cdots, f)$, where $e, f$ are idempotent matrices with entries in $(\mathcal{R}(\Gamma, \sigma))^{\sim}=$ unital algebra obtained by adding the identity to $\mathcal{R}(\Gamma, \sigma)$ and $\widetilde{\operatorname{tr}}_{c}$ denotes the canonical extension of $\operatorname{tr}_{c}$ to $(\mathcal{R}(\Gamma, \sigma))^{\sim}$. Let $\widetilde{\not_{\mathcal{E}}^{+}} \otimes \nabla$ be the Dirac operator defined in the 
previous section, which is invariant under the projective action of the fundamental group defined by $\sigma$. Recall that by definition, the $(c, \Gamma, \sigma)$-index of $\widetilde{\partial_{\mathcal{E}}^{+}} \otimes \nabla$ is

$$
\operatorname{Ind}_{(c, \Gamma, \sigma)}\left(\widetilde{\partial_{\mathcal{E}}^{+}} \otimes \nabla\right)=\left[\operatorname{tr}_{c}\right]\left(\operatorname{Ind}_{\sigma}\left(\widetilde{\not_{\mathcal{E}}^{+}} \otimes \nabla\right)\right)=\left\langle\left[\operatorname{tr}_{c}\right], \mu_{\sigma}([\mathcal{E}])\right\rangle \in \mathbb{R} .
$$

It only depends on the cohomology class $[c] \in H^{2}(\Gamma)$, and it is linear with respect to $[c]$. We assemble this to give the following theorem.

Theorem 3.1. Given $[c] \in H^{2}(\Gamma)$ and $\sigma \in H^{2}(\Gamma, U(1))$ a multiplier on $\Gamma$, there is a canonical additive map

$$
\langle[c], \quad\rangle: K_{\text {orb }}^{0}\left(\Sigma\left(g ; \nu_{1}, \ldots, \nu_{n}\right)\right) \rightarrow \mathbb{R}
$$

which is defined as

$$
\langle[c],[\mathcal{E}]\rangle=\operatorname{Ind}_{(c, \Gamma, \sigma)}\left(\widetilde{\partial_{\mathcal{E}}^{+}} \otimes \nabla\right)=\left[\operatorname{tr}_{c}\right]\left(\operatorname{Ind}_{\sigma}\left(\widetilde{\partial_{\mathcal{E}}^{+}} \otimes \nabla\right)\right)=\left\langle\left[\operatorname{tr}_{c}\right], \mu_{\sigma}([\mathcal{E}])\right\rangle \in \mathbb{R} .
$$

Moreover, it is linear with respect to $[c]$.

The area cocycle $c$ of the Fuchsian group $\Gamma$ is a canonically defined 2-cocycle on $\Gamma$ that is defined as follows. Firstly, recall that there is a well known area 2-cocycle on $P S L(2, \mathbb{R})$, cf. [Co2, defined as follows: $P S L(2, \mathbb{R})$ acts on $\mathbb{H}$ such that $\mathbb{H} \cong P S L(2, \mathbb{R}) / S O(2)$. Then $c\left(g_{1}, g_{2}\right)=$ Area $\left(\Delta\left(o, g_{1} . o, g_{2}{ }^{-1} . o\right)\right) \in \mathbb{R}$, where $o$ denotes an origin in $\mathbb{H}$ and Area $(\Delta(a, b, c))$ denotes the hyperbolic area of the geodesic triangle in $\mathbb{H}$ with vertices at $a, b, c \in \mathbb{H}$. Then the restriction of $c$ to the subgroup $\Gamma$ is the area cocycle $c$ of $\Gamma$.

Corollary 3.2. Let $c,[c] \in H^{2}(\Gamma)$, be the area cocycle, and $\mathcal{E} \rightarrow \Sigma\left(g ; \nu_{1}, \ldots, \nu_{n}\right)$ be an orbifold vector bundle over the orbifold $\Sigma\left(g ; \nu_{1}, \ldots, \nu_{n}\right)$. Then in the notation above, one has

$$
\langle[c],[\mathcal{E}]\rangle=\phi \operatorname{rank} \mathcal{E} \in \phi \mathbb{Z} .
$$

where $-\phi=2(1-g)+(\nu-n) \in \mathbb{Q}$ is the orbifold Euler characteristic of $\Sigma\left(g ; \nu_{1}, \ldots, \nu_{n}\right)$ and $\nu=\sum_{j=1}^{n} 1 / \nu_{j}$.

Proof. By Theorem 2.2, one has one has

$$
\left[\operatorname{tr}_{c}\right]\left(\operatorname{Ind}_{\sigma}\left(\widetilde{\partial_{\mathcal{E}}^{+}} \otimes \nabla\right)\right)=\frac{1}{2 \pi \#(G)} \int_{\Sigma_{g^{\prime}}} \hat{A}(\Omega) \operatorname{tr}\left(e^{R^{\mathcal{E}}}\right) e^{\omega} \psi^{*}(\tilde{c}),
$$

where $\Sigma_{g^{\prime}}$ is smooth and $G \rightarrow \Sigma_{g^{\prime}} \rightarrow \Sigma\left(g ; \nu_{1}, \ldots, \nu_{n}\right)$ is a finite orbifold cover. Here $\psi: \Sigma_{g^{\prime}} \rightarrow \Sigma_{g^{\prime}}$ is the lift of the map $f: \Sigma\left(g ; \nu_{1}, \ldots \nu_{n}\right) \rightarrow \Sigma\left(g ; \nu_{1}, \ldots \nu_{n}\right)$ (since $\underline{B} \Gamma=\Sigma\left(g ; \nu_{1}, \ldots \nu_{n}\right)$ in this case) which is the classifying map of the orbifold universal cover (and which in this case is the identity map) and $[\tilde{c}]$ degree 2 cohomology class on $\Sigma_{g^{\prime}}$ that is the lift of $c$ to $\Sigma_{g^{\prime}}$. We next simplify the right hand side of (7) using the fact that $\hat{A}(\Omega)=1$ and that

$$
\begin{aligned}
\operatorname{tr}\left(e^{R^{\mathcal{E}}}\right) & =\operatorname{rank} \mathcal{E}+\operatorname{tr}\left(R^{\mathcal{E}}\right), \\
\psi^{*}(\tilde{c}) & =\tilde{c}, \\
e^{\omega} & =1+\omega .
\end{aligned}
$$

We obtain

$$
\left[\operatorname{tr}_{c}\right]\left(\operatorname{Ind}_{\sigma}\left(\widetilde{\partial_{\mathcal{E}}^{+}} \otimes \nabla\right)\right)=\frac{\operatorname{rank} \mathcal{E}}{2 \pi \#(G)}\left\langle[\tilde{c}],\left[\Sigma_{g^{\prime}}\right]\right\rangle
$$


When $c,[c] \in H^{2}(\Gamma)$, is the area 2-cocycle, then $\tilde{c}$ is merely the restriction of the area cocycle on $P S L(2, \mathbb{R})$ to the subgroup $\Gamma_{g^{\prime}}$. Then one has

$$
\left\langle[\tilde{c}],\left[\Sigma_{g^{\prime}}\right]\right\rangle=-2 \pi \chi\left(\Sigma_{g^{\prime}}\right)=4 \pi\left(g^{\prime}-1\right) .
$$

The corollary now follows from Theorem 3.1 above together with the fact that $g^{\prime}=1+\frac{\#(G)}{2}(2(g-$ 1) $+(n-\nu))$, and $\nu=\sum_{j=1}^{n} 1 / \nu_{j}$.

We next describe the canonical pairing of $K_{0}\left(C_{r}^{*}(\Gamma, \sigma)\right)$, given $[c] \in H^{2}(\Gamma)$. Since $\Sigma\left(g ; \nu_{1}, \ldots, \nu_{n}\right)$ is negatively curved, we know from [Ji] that

$$
\mathcal{R}(\Gamma, \sigma)=\left\{f:\left.\Gamma \rightarrow \mathbb{C}\left|\sum_{\gamma \in \Gamma}\right| f(\gamma)\right|^{2}(1+l(\gamma))^{k}<\infty \text { for all } k \geq 0\right\},
$$

where $l: \Gamma \rightarrow \mathbb{R}^{+}$denotes the length function, is a dense and spectral invariant subalgebra of $C_{r}^{*}(\Gamma, \sigma)$. In particular it is closed under the smooth functional calculus, and is known as the algebra of rapidly decreasing $L^{2}$ functions on $\Gamma$. By a theorem of Bost, the inclusion map $\mathcal{R}(\Gamma, \sigma) \subset C_{r}^{*}(\Gamma, \sigma)$ induces an isomorphism

$$
K_{j}(\mathcal{R}(\Gamma, \sigma)) \cong K_{j}\left(C_{r}^{*}(\Gamma, \sigma)\right), \quad j=0,1 .
$$

The desired pairing is the one obtained from the canonical pairing of $K_{0}(\mathcal{R}(\Gamma, \sigma))$ with $[c] \in H^{2}(\Gamma)$ using the canonical isomorphism. Therefore one has the equality

$$
\left\langle[c], \mu_{\sigma}^{-1}[P]\right\rangle=\left\langle\left[\operatorname{tr}_{c}\right],[P]\right\rangle
$$

for any $[P] \in K_{0}(\mathcal{R}(\Gamma, \sigma)) \cong K_{0}\left(C_{r}^{*}(\Gamma, \sigma)\right)$. Using the previous corollary, one has

Theorem 3.3 (Range of the higher trace on $K$-theory). Let $c$ be the area 2-cocycle on $\Gamma$. Then $c$ is known to be a bounded 2-cocycle, and one has

$$
\left\langle\left[\operatorname{tr}_{c}\right],[P]\right\rangle=\phi\left(\operatorname{rank} \mathcal{E}^{0}-\operatorname{rank} \mathcal{E}^{1}\right) \in \phi \mathbb{Z}
$$

where $-\phi=2(1-g)+(\nu-n) \in \mathbb{Q}$ is the orbifold Euler characteristic of $\Sigma\left(g ; \nu_{1}, \ldots, \nu_{n}\right)$ and $\nu=\sum_{j=1}^{n} 1 / \nu_{j}$. Here $[P] \in K_{0}(\mathcal{R}(\Gamma, \sigma)) \cong K_{0}\left(C_{r}^{*}(\Gamma, \sigma)\right)$, and $\mathcal{E}^{0}, \mathcal{E}^{1}$ are orbifold vector bundles over $\Sigma\left(g ; \nu_{1}, \ldots, \nu_{n}\right)$ such that

$$
\mu_{\sigma}^{-1}([P])=\left[\mathcal{E}^{0}\right]-\left[\mathcal{E}^{1}\right] \in K_{\text {orb }}^{0}\left(\Sigma\left(g ; \nu_{1}, \ldots, \nu_{n}\right)\right) .
$$

In particular, the range of the the higher trace on $K$-theory is

$$
\left[\operatorname{tr}_{c}\right]\left(K_{0}\left(C^{*}(\Gamma, \sigma)\right)\right)=\phi \mathbb{Z} .
$$

Note that $\phi$ is in general only a rational number and we will give examples to show that this is the case; however it is an integer whenever the orbifold is smooth, i.e. whenever $1=\nu_{1}=\ldots=\nu_{n}$, which is the case considered in [CHMM]. We will apply this result in the next section to compute the range of values the Hall conductance in the quantum Hall effect on the hyperbolic plane, for orbifold fundamental groups, extending the results in CHMM.

In the last section we provide a list of specific examples where fractional values are achieved, and discuss the physical significance of our model. 


\section{The Area cocycle, the hyperbolic Connes-Kubo formula and the Quantum HALL EFFECT}

In this section, we adapt and generalize the discrete model of the quantum Hall effect of Bellissard and his collaborators Bel+E+S] and also [CHMM, to the case of general cocompact Fuchsian groups and orbifolds, which can be viewed equivalently as the generalization to the equivariant context. We will first derive the discrete analogue of the hyperbolic Connes-Kubo formula for the Hall conductance 2-cocycle, which was derived in the continuous case in [CHMM]. We then relate it to the Area 2-cocycle on the twisted group algebra of the discrete Fuchsian group, and we show that these define the same cyclic cohomology class. This enables us to use the results of the previous section to show that the Hall conductance has plateaux at all energy levels belonging to any gap in the spectrum of the Hamiltonian, where it is now shown to be equal to an integral multiple of a fractional valued topological invariant, namely the orbifold Euler characteristic. The presence of denominators is caused by the presence of cone points singularities and by the hyperbolic geometry on the complement of these cone points. Moreover the set of possible denominators is finite and has been explicitly determined in the next section, and the results compared to the experimental data. It is plausible that this might shed light on the mathematical mechanism responsible for fractional quantum numbers in the quantum Hall effect.

We consider the Cayley graph of the Fuchsian group $\Gamma$ of signature $\left(g ; \nu_{1}, \ldots, \nu_{n}\right)$, which acts freely on the complement of a countable set of points in the hyperbolic plane. The Cayley graph embeds in the hyperbolic plane as follows. Fix a base point $u \in \mathbb{H}$ such that the stabilizer (or isotropy subgroup) at $u$ is trivial and consider the orbit of the $\Gamma$ action through $u$. This gives the vertices of the graph. The edges of the graph are geodesics constructed as follows. Each element of the group $\Gamma$ may be written as a word of minimal length in the generators of $\Gamma$ and their inverses. Each generator and its inverse determine a unique geodesic emanating from a vertex $x$ and these geodesics form the edges of the graph. Thus each word $x$ in the generators determines a piecewise geodesic path from $u$ to $x$.

Recall that the area cocycle $c$ of the Fuchsian group $\Gamma$ is a canonically defined 2-cocycle on $\Gamma$ that is defined as follows. Firstly, recall that there is a well known area 2-cocycle on $P S L(2, \mathbb{R})$, cf. Co2 , defined as follows: $P S L(2, \mathbb{R})$ acts on $\mathbb{H}$ such that $\mathbb{H} \cong P S L(2, \mathbb{R}) / S O(2)$. Then $c\left(\gamma_{1}, \gamma_{2}\right)=\operatorname{Area}\left(\Delta\left(o, \gamma_{1} . o, \gamma_{2}^{-1} . o\right)\right) \in \mathbb{R}$, where $o$ denotes an origin in $\mathbb{H}$ and $\operatorname{Area}(\Delta(a, b, c))$ denotes the hyperbolic area of the geodesic triangle in $\mathbb{H}$ with vertices at $a, b, c \in \mathbb{H}$. Then the restriction of $c$ to the subgroup $\Gamma$ is the area cocycle $c$ of $\Gamma$.

This area cocycle defines in a canonical way a cyclic 2 -cocycle $\operatorname{tr}_{c}$ on the group algebra $\mathbb{C}(\Gamma, \sigma)$ as follows;

$$
\operatorname{tr}_{c}\left(a_{0}, a_{1}, a_{2}\right)=\sum_{\gamma_{0} \gamma_{1} \gamma_{2}=1} a_{0}\left(\gamma_{0}\right) a_{1}\left(\gamma_{1}\right) a_{2}\left(\gamma_{2}\right) c\left(\gamma_{1}, \gamma_{2}\right) \sigma\left(\gamma_{1}, \gamma_{2}\right)
$$

We will now describe the hyperbolic Connes-Kubo formula for the Hall conductance in the Quantum Hall Effect. Let $\Omega_{j}$ denote the (diagonal) operator on $\ell^{2}(\Gamma)$ defined by

$$
\Omega_{j} f(\gamma)=\Omega_{j}(\gamma) f(\gamma) \quad \forall f \in \ell^{2}(\Gamma) \quad \forall \gamma \in \Gamma
$$


where

and where

$$
\Omega_{j}(\gamma)=\int_{o}^{\gamma \cdot o} \alpha_{j} \quad j=1, \ldots, 2 g
$$

$$
\left\{\alpha_{j}\right\}_{j=1, \ldots, 2 g}=\left\{a_{j}\right\}_{j=1, \ldots, g} \cup\left\{b_{j}\right\}_{j=1, \ldots, g}
$$

is a collection of harmonic $V$-forms on the orbifold $\Sigma\left(g ; \nu_{1}, \ldots, \nu_{n}\right)$, generating $H^{1}\left(\Sigma_{g}, \mathbb{R}\right)=\mathbb{R}^{2 g}$, cf. Kaw2 pg.78-83. These correspond to harmonic $G$-invariant forms on $\Sigma_{g^{\prime}}$ and to harmonic $\Gamma$-invariant forms on $\mathbb{H}$.

Notice that we can write equivalently

$$
\Omega_{j}(\gamma)=c_{j}(\gamma)
$$

where the group cocycles $c_{j}$ form a symplectic basis for $H^{1}(\Gamma, \mathbb{Z})=\mathbb{Z}^{2 g}$, with generators $\left\{\alpha_{j}\right\}_{j=1, \ldots, 2 g}$, as in (9) and can be defined as the integration on loops on the Riemann surface of genus $g$ underlying the orbifold $\Sigma\left(g ; \nu_{1}, \ldots, \nu_{n}\right)$,

$$
c_{j}(\gamma)=\int_{\gamma} \alpha_{j}
$$

For $j=1, \ldots, 2 g$, define the derivations $\delta_{j}$ on $\mathcal{R}(\Gamma, \sigma)$ as being the commutators $\delta_{j} a=\left[\Omega_{j}, a\right]$. A simple calculation shows that

$$
\delta_{j} a(\gamma)=\Omega_{j}(\gamma) a(\gamma) \quad \forall a \in \mathcal{R}(\Gamma, \sigma) \quad \forall \gamma \in \Gamma .
$$

Thus, we can view this as the following general construction. Given a 1-cocycle $a$ on the discrete group $\Gamma$, i.e.

$$
a\left(\gamma_{1} \gamma_{2}\right)=a\left(\gamma_{1}\right)+a\left(\gamma_{2}\right) \quad \forall \gamma_{1}, \gamma_{2} \in \Gamma
$$

one can define a derivation $\delta_{a}$ on the twisted group algebra $\mathbb{C}(\Gamma, \sigma)$

$$
\delta_{a}(f)(\gamma)=a(\gamma) f(\gamma)
$$

Then we verify that

$$
\begin{aligned}
\delta_{a}(f g)(\gamma) & =a(\gamma) f g(\gamma) \\
& =a(\gamma) \sum_{\gamma=\gamma_{1} \gamma_{2}} f\left(\gamma_{1}\right) g\left(\gamma_{2}\right) \sigma\left(\gamma_{1}, \gamma_{2}\right) \\
& =\sum_{\gamma=\gamma_{1} \gamma_{2}}\left(a\left(\gamma_{1}\right)+a\left(\gamma_{2}\right)\right) f\left(\gamma_{1}\right) g\left(\gamma_{2}\right) \sigma\left(\gamma_{1}, \gamma_{2}\right) \\
& =\sum_{\gamma=\gamma_{1} \gamma_{2}}\left(\delta_{a}(f)\left(\gamma_{1}\right) g\left(\gamma_{2}\right) \sigma\left(\gamma_{1}, \gamma_{2}\right)+f\left(\gamma_{1}\right) \delta_{a}(g)\left(\gamma_{2}\right) \sigma\left(\gamma_{1}, \gamma_{2}\right)\right) \\
& =\left(\delta_{a}(f) g\right)(\gamma)+\left(f \delta_{a} g\right)(\gamma) .
\end{aligned}
$$

As determined in section 1 , the first cohomology of the group $\Gamma=\Gamma\left(g ; \nu_{1}, \ldots, \nu_{n}\right)$ is a free Abelian group of rank $2 g$. It is in fact a symplectic vector space over $\mathbb{Z}$, and assume that 
$a_{j}, b_{j}, j=1, \ldots g$ is a symplectic basis of $H^{1}(\Gamma, \mathbb{Z})$, as in (9). We denote $\delta_{a_{j}}$ by $\delta_{j}$ and $\delta_{b_{j}}$ by $\delta_{j+g}$. Then these derivations give rise to cyclic 2-cocycle on the twisted group algebra $\mathbb{C}(\Gamma, \sigma)$,

$$
\operatorname{tr}^{K}\left(f_{0}, f_{1}, f_{2}\right)=\sum_{j=1}^{g} \operatorname{tr}\left(f_{0}\left(\delta_{j}\left(f_{1}\right) \delta_{j+g}\left(f_{2}\right)-\delta_{j+g}\left(f_{1}\right) \delta_{j}\left(f_{2}\right)\right)\right)
$$

$\operatorname{tr}^{K}$ is called the Connes-Kubo Hall conductance cyclic 2-cocycle.

In terms of the $\Omega_{j}$, note that we have the simple estimate

$$
\left|\Omega_{j}(\gamma)\right| \leq\left\|a_{j}\right\|_{(\infty)} d(\gamma . o, o)
$$

where $d(\gamma . o, o)$ and the distance $d_{\Gamma}(\gamma, 1)$ in the word metric on the group $\Gamma$ are equivalent. This then yields the estimate

$$
\left|\delta_{j} a(\gamma)\right| \leq C_{N} d_{\Gamma}(\gamma, 1)^{-N} \quad \forall N \in \mathbb{N}
$$

i.e $\delta_{j} a \in \mathcal{R}(\Gamma, \sigma) \quad \forall a \in \mathcal{R}(\Gamma, \sigma)$. Note that since $\forall \gamma, \gamma^{\prime} \in \Gamma$, the difference $\Omega_{j}\left(\gamma \gamma^{\prime}\right)-\Omega_{j}\left(\gamma^{\prime}\right)$ is a constant independent of $\gamma^{\prime}$, we see that $\Gamma$-equivariance is preserved. For $j=1, \ldots, 2 g$, define the cyclic 2-cocycles

$$
\operatorname{tr}_{j}^{K}\left(a_{0}, a_{1}, a_{2}\right)=\operatorname{tr}\left(a_{0}\left(\delta_{j} a_{1} \delta_{j+g} a_{2}-\delta_{j+g} a_{1} \delta_{j} a_{2}\right)\right) .
$$

These compute the Hall conductance for currents in the $(j+g)$ th direction which are induced by electric fields in the $j$ th direction, as can be shown using the quantum adiabatic theorem of Avron-Seiler-Yaffe Av+S+Y just as in section 6 of [CHMM, in the continuous model. Then the hyperbolic Connes-Kubo formula for the Hall conductance is the cyclic 2-cocycle given by the sum

$$
\operatorname{tr}^{K}\left(a_{0}, a_{1}, a_{2}\right)=\sum_{j=1}^{g} \operatorname{tr}_{j}^{K}\left(a_{0}, a_{1}, a_{2}\right) .
$$

Theorem 4.1 (The Comparison Theorem).

$$
\left[\operatorname{tr}^{K}\right]=\left[\operatorname{tr}_{c}\right] \in H C^{2}(\mathcal{R}(\Gamma, \sigma))
$$

Proof: Our aim is now to compare the two cyclic 2-cocycles and to prove that they differ by a coboundary i.e.

$$
\operatorname{tr}^{K}\left(a_{0}, a_{1}, a_{2}\right)-\operatorname{tr}_{c}\left(a_{0}, a_{1}, a_{2}\right)=b \lambda\left(a_{0}, a_{1}, a_{2}\right)
$$

for some cyclic 1-cochain $\lambda$ and where $b$ is the cyclic coboundary operator. The key to this theorem is a geometric interpretation of the hyperbolic Connes-Kubo formula.

We begin with some calculations, to enable us to make this comparison of the cyclic 2-cocycles.

$$
\begin{gathered}
\operatorname{tr}^{K}\left(a_{0}, a_{1}, a_{2}\right)= \\
\sum_{j=1}^{g} \sum_{\gamma_{0} \gamma_{1} \gamma_{2}=1} a_{0}\left(\gamma_{0}\right)\left(\delta_{j} a_{1}\left(\gamma_{1}\right) \delta_{j+g} a_{2}\left(\gamma_{2}\right)-\delta_{j+g} a_{1}\left(\gamma_{1}\right) \delta_{j} a_{2}\left(\gamma_{2}\right)\right) \sigma\left(\gamma_{0}, \gamma_{1}\right) \sigma\left(\gamma_{0} \gamma_{1}, \gamma_{2}\right) \\
=\sum_{j=1}^{g} \sum_{\gamma_{0} \gamma_{1} \gamma_{2}=1} a_{0}\left(\gamma_{0}\right) a_{1}\left(\gamma_{1}\right) a_{2}\left(\gamma_{2}\right)\left(\Omega_{j}\left(\gamma_{1}\right) \Omega_{j+g}\left(\gamma_{2}\right)-\Omega_{j+g}\left(\gamma_{1}\right) \Omega_{j}\left(\gamma_{2}\right)\right) \sigma\left(\gamma_{1}, \gamma_{2}\right)
\end{gathered}
$$


since by the cocycle identity for multipliers, one has

$$
\begin{aligned}
\sigma\left(\gamma_{0}, \gamma_{1}\right) \sigma\left(\gamma_{0} \gamma_{1}, \gamma_{2}\right) & =\sigma\left(\gamma_{0}, \gamma_{1} \gamma_{2}\right) \sigma\left(\gamma_{1}, \gamma_{2}\right) \\
& =\sigma\left(\gamma_{0}, \gamma_{0}^{-1}\right) \sigma\left(\gamma_{1}, \gamma_{2}\right) \quad \text { since } \quad \gamma_{0} \gamma_{1} \gamma_{2}=1 \\
& =\sigma\left(\gamma_{1}, \gamma_{2}\right) \quad \text { since } \sigma\left(\gamma_{0}, \gamma_{0}^{-1}\right)=1 .
\end{aligned}
$$

So we are now in a position to compare the two cyclic 2-cocycles. Define $\Psi_{j}\left(\gamma_{1}, \gamma_{2}\right)=\Omega_{j}\left(\gamma_{1}\right) \Omega_{j+g}\left(\gamma_{2}\right)-$ $\Omega_{j+g}\left(\gamma_{1}\right) \Omega_{j}\left(\gamma_{2}\right)$.

Let $\Xi: \mathbb{H} \rightarrow \mathbb{R}^{2 g}$ denote the Abel-Jacobi map

$$
\Xi: x \mapsto\left(\int_{o}^{x} a_{1}, \int_{o}^{x} b_{1}, \ldots, \int_{o}^{x} a_{g}, \int_{o}^{x} b_{g}\right),
$$

where $\int_{o}^{x}$ means integration along the unique geodesic in $\mathbb{H}$ connecting $o$ to $x$. The origin $o$ is chosen so that it satisfies $\Gamma . o \cong \Gamma$. The map $\Xi$ is a symplectic map, that is, if $\omega$ and $\omega_{J}$ are the respective symplectic 2 -forms, then one has $\Xi^{*}\left(\omega_{J}\right)=\omega$. One then has the following geometric lemma.

\section{Lemma 4.2.}

$$
\sum_{j=1}^{g} \Psi_{j}\left(\gamma_{1}, \gamma_{2}\right)=\int_{\Delta_{E}\left(\gamma_{1}, \gamma_{2}\right)} \omega_{J}
$$

where $\Delta_{E}\left(\gamma_{1}, \gamma_{2}\right)$ denotes the Euclidean triangle with vertices at $\Xi(o), \Xi\left(\gamma_{1} . o\right)$ and $\Xi\left(\gamma_{2} . o\right)$, and $\omega_{J}$ denotes the flat Kähler 2-form on the Jacobi variety. That is, $\sum_{j=1}^{g} \Psi_{j}\left(\gamma_{1}, \gamma_{2}\right)$ is equal to the Euclidean area of the Euclidean triangle $\Delta_{E}\left(\gamma_{1}, \gamma_{2}\right)$.

Proof. We need to consider the expression

$$
\sum_{j=1}^{g} \Psi_{j}\left(\gamma_{1}, \gamma_{2}\right)=\sum_{j=1}^{g} \Omega_{j}\left(\gamma_{1}\right) \Omega_{j+g}\left(\gamma_{2}\right)-\Omega_{j+g}\left(\gamma_{1}\right) \Omega_{j}\left(\gamma_{2}\right) .
$$

Let $s$ denote the symplectic form on $\mathbb{R}^{2 g}$ given by:

$$
s(u, v)=\sum_{j=1}^{g}\left(u_{j} v_{j+g}-u_{j+g} v_{j}\right) .
$$

The so-called 'symplectic area' of a triangle with vertices $\Xi(o)=0, \Xi\left(\gamma_{1} . o\right), \Xi\left(\gamma_{2} . o\right)$ may be seen to be $s\left(\Xi\left(\gamma_{1} . o\right), \Xi\left(\gamma_{2} . o\right)\right)$. To appreciate this, however, we need to use an argument from [GH], pages 333 -336. In terms of the standard basis of $\mathbb{R}^{2 g}$ (given in this case by vertices in the integer period lattice arising from our choice of basis of harmonic one forms) and corresponding coordinates $u_{1}, u_{2}, \ldots u_{2 g}$ the form $s$ is the two form on $\mathbb{R}^{2 g}$ given by

$$
\omega_{J}=\sum_{j=1}^{g} d u_{j} \wedge d u_{j+g} .
$$

Now the 'symplectic area' of a triangle in $\mathbb{R}^{2 g}$ with vertices $\Xi(o)=0, \Xi\left(\gamma_{1} . o\right), \Xi\left(\gamma_{2} . o\right)$ is given by integrating $\omega_{J}$ over the triangle and a brief calculation reveals that this yields $s\left(\Xi\left(\gamma_{1} . o\right), \Xi\left(\gamma_{2} . o\right)\right) / 2$, proving the lemma. 
We also observe that since $\omega=\Xi^{*} \omega_{J}$, one has

$$
c\left(\gamma_{1}, \gamma_{2}\right)=\int_{\Delta\left(\gamma_{1}, \gamma_{2}\right)} \omega=\int_{\Xi\left(\Delta\left(\gamma_{1}, \gamma_{2}\right)\right)} \omega_{J}
$$

Therefore the difference

$$
\begin{aligned}
\sum_{j=1}^{g} \Psi_{j}\left(\gamma_{1}, \gamma_{2}\right)-c\left(\gamma_{1}, \gamma_{2}\right) & =\int_{\Delta_{E}\left(\gamma_{1}, \gamma_{2}\right)} \omega_{J}-\int_{\Xi\left(\Delta\left(\gamma_{1}, \gamma_{2}\right)\right)} \omega_{J} \\
& =\int_{\partial \Delta_{E}\left(\gamma_{1}, \gamma_{2}\right)} \Theta_{J}-\int_{\partial \Xi\left(\Delta\left(\gamma_{1}, \gamma_{2}\right)\right)} \Theta_{J}
\end{aligned}
$$

where $\Theta_{J}$ is a 1-form on the universal cover of the Jacobi variety such that $d \Theta_{J}=\omega_{J}$. Therefore one has

$$
\sum_{j=1}^{g} \Psi_{j}\left(\gamma_{1}, \gamma_{2}\right)-c\left(\gamma_{1}, \gamma_{2}\right)=h\left(1, \gamma_{1}\right)-h\left(\gamma_{1}^{-1}, \gamma_{2}\right)+h\left(\gamma_{2}^{-1}, 1\right)
$$

where $h\left(\gamma_{1}^{-1}, \gamma_{2}\right)=\int_{\Xi\left(\ell\left(\gamma_{1}, \gamma_{2}\right)\right)} \Theta_{J}-\int_{m\left(\gamma_{1}, \gamma_{2}\right)} \Theta_{J}$, where $\ell\left(\gamma_{1}, \gamma_{2}\right)$ denotes the unique geodesic in $\mathbb{H}$ joining $\gamma_{1} . o$ and $\gamma_{2} . o$ and $m\left(\gamma_{1}, \gamma_{2}\right)$ is the straight line in the Jacobi variety joining the points $\Xi\left(\gamma_{1} . o\right)$ and $\Xi\left(\gamma_{2} . o\right)$. Since we can also write $h\left(\gamma_{1}^{-1}, \gamma_{2}\right)=\int_{D\left(\gamma_{1}, \gamma_{2}\right)} \omega_{J}$, where $D\left(\gamma_{1}, \gamma_{2}\right)$ is a disk in the Jacobi variety with boundary $\Xi\left(\ell\left(\gamma_{1}, \gamma_{2}\right)\right) \cup m\left(\gamma_{1}, \gamma_{2}\right)$, we see that $h$ is $\Gamma$-invariant.

We now define the cyclic 1-cochain $\lambda$ on $\mathcal{R}(\Gamma, \sigma)$ as

$$
\lambda\left(a_{0}, a_{1}\right)=\operatorname{tr}\left(\left(a_{0}\right)_{h} a_{1}\right)=\sum_{\gamma_{0} \gamma_{1}=1} h\left(1, \gamma_{1}\right) a_{0}\left(\gamma_{0}\right) a_{1}\left(\gamma_{1}\right) \sigma\left(\gamma_{0}, \sigma_{1}\right)
$$

where $\left(a_{0}\right)_{h}$ is the operator on $\ell^{2}(\Gamma)$ whose matrix in the canonical basis is $h\left(\gamma_{1}, \gamma_{2}\right) a_{0}\left(\gamma_{1} \gamma_{2}^{-1}\right)$. Firstly, one has by definition

$$
b \lambda\left(a_{0}, a_{1}, a_{2}\right)=\lambda\left(a_{0} a_{1}, a_{2}\right)-\lambda\left(a_{0}, a_{1} a_{2}\right)+\lambda\left(a_{2} a_{0}, a_{1}\right)
$$

We compute each of the terms seperately

$$
\begin{aligned}
& \lambda\left(a_{0} a_{1}, a_{2}\right)=\sum_{\gamma_{0} \gamma_{1} \gamma_{2}=1} h\left(1, \gamma_{2}\right) a_{0}\left(\gamma_{0}\right) a_{1}\left(\gamma_{1}\right) a_{2}\left(\gamma_{2}\right) \sigma\left(\gamma_{1}, \gamma_{2}\right) \\
& \lambda\left(a_{0}, a_{1} a_{2}\right)=\sum_{\gamma_{0} \gamma_{1} \gamma_{2}=1} h\left(1, \gamma_{1} \gamma_{2}\right) a_{0}\left(\gamma_{0}\right) a_{1}\left(\gamma_{1}\right) a_{2}\left(\gamma_{2}\right) \sigma\left(\gamma_{1}, \gamma_{2}\right) \\
& \lambda\left(a_{2} a_{0}, a_{1}\right)=\sum_{\gamma_{0} \gamma_{1} \gamma_{2}=1} h\left(1, \gamma_{1}\right) a_{0}\left(\gamma_{0}\right) a_{1}\left(\gamma_{1}\right) a_{2}\left(\gamma_{2}\right) \sigma\left(\gamma_{1}, \gamma_{2}\right)
\end{aligned}
$$

Now by $\Gamma$-equivariance, $h\left(1, \gamma_{1} \gamma_{2}\right)=h\left(\gamma_{1}^{-1}, \gamma_{2}\right)$ and $h\left(1, \gamma_{2}\right)=h\left(\gamma_{2}^{-1}, 1\right)$. Therefore one has

$$
\begin{gathered}
b \lambda\left(a_{0}, a_{1}, a_{2}\right)= \\
\sum_{\gamma_{0} \gamma_{1} \gamma_{2}=1} a_{0}\left(\gamma_{0}\right) a_{1}\left(\gamma_{1}\right) a_{2}\left(\gamma_{2}\right)\left(h\left(\gamma_{2}^{-1}, 1\right)-h\left(\gamma_{1}^{-1}, \gamma_{2}\right)+h\left(1, \gamma_{1}\right)\right) \sigma\left(\gamma_{1}, \gamma_{2}\right)
\end{gathered}
$$

Using the formula above, we see that

$$
b \lambda\left(a_{0}, a_{1}, a_{2}\right)=\operatorname{tr}^{K}\left(a_{0}, a_{1}, a_{2}\right)-\operatorname{tr}_{c}\left(a_{0}, a_{1}, a_{2}\right) .
$$


It follows from Connes pairing theory of cyclic cohomology and $K$-theory [Co2], by the range of the higher trace Theorem 3.3 and by the Comparison Theorem 4.1 above that

Corollary 4.3 (Rationality of conductance). The Connes-Kubo Hall conductance cocycle $\operatorname{tr}^{K}$ is rational. More precisely, one has

$$
\operatorname{tr}^{K}(P, P, P)=\operatorname{tr}_{c}(P, P, P) \in \phi \mathbb{Z}
$$

for all projections $P \in \mathcal{R}(\Gamma, \sigma)$, where $-\phi=2(1-g)+(\nu-n) \in \mathbb{Q}$ is the orbifold Euler characteristic of $\Sigma\left(g ; \nu_{1}, \ldots, \nu_{n}\right)$.

Finally, suppose that we are given a very thin sample of pure metal, with electrons situated along the Cayley graph of $\Gamma$, and a very strong magnetic field which is uniform and normal in direction to the sample. Then at very low temperatures, close to absolute zero, quantum mechanics dominates and the discrete model that is considered here is a model of electrons moving on the Cayley graph of $\Gamma$ which is embedded in the sample. The associated discrete Hamiltonian $H_{\sigma}$ for the electron in the magnetic field is given by the Random Walk operator in the projective $(\Gamma, \sigma)$ regular representation on the Cayley graph of the group $\Gamma$. It is also known as the generalized Harper operator and was first studied in this generalized context in [Sun, see also [CHMM]. We will see that the Hamiltonian that we consider is in a natural way the sum of a free Hamiltonian and a term that models the Coulomb interaction. We also add a restricted class of potential terms to the Hamiltonian in our model.

Because the charge carriers are Fermions, two different charge carriers must occupy different quantum eigenstates of the Hamiltonian. In the limit of zero temperature they minimize the energy and occupy eigenstates with energy lower that a given one, called the Fermi level and denoted $E$. Let $P_{E}$ denote denote the corresponding spectral projection of the Hamiltonian. If $E$ is not in the spectrum of the Hamiltonian, then then $P_{E} \in \mathcal{R}(\Gamma, \sigma)$ and the hyperbolic Connes-Kubo formula for the Hall conductance $\sigma_{E}$ at the energy level $E$ is defined as follows;

$$
\sigma_{E}=\operatorname{tr}^{K}\left(P_{E}, P_{E}, P_{E}\right) .
$$

As mentioned earlier, it measures the sum of the contributions to the Hall conductance at the energy level $E$ for currents in the $(j+g)$ th direction which are induced by electric fields in the $j$ th direction, cf. section 6 [CHMM. By Corollary 4.3, one knows that the Hall conductance takes on values in $\phi \mathbb{Z}$ whenever the energy level $E$ lies in a gap in the spectrum of the Hamiltonian $H_{\sigma}$. In fact we notice that the Hall conductance is a constant function of the energy level $E$ for all values of $E$ in the same gap in the spectrum of the Hamiltonian. That is, the Hall conductance has plateaux which are integer multiples of the fraction $\phi$ on the gap in the spectrum of the Hamiltonian.

We now give some details. Recall the left $\sigma$-regular representation

$$
(U(\gamma) f)\left(\gamma^{\prime}\right)=f\left(\gamma^{-1} \gamma^{\prime}\right) \sigma\left(\gamma^{\prime}, \gamma^{-1} \gamma^{\prime}\right)
$$

$\forall f \in \ell^{2}(\Gamma)$ and $\forall \gamma, \gamma^{\prime} \in \Gamma$. It has the property that

$$
U(\gamma) U\left(\gamma^{\prime}\right)=\sigma\left(\gamma, \gamma^{\prime}\right) U\left(\gamma \gamma^{\prime}\right)
$$


Let $S=\left\{A_{j}, B_{j}, A_{j}^{-1}, B_{j}^{-1}, C_{i}, C_{i}^{-1}: j=1, \ldots, g, \quad i=1, \ldots, n\right\}$ be a symmetric set of generators for $\Gamma$. Then the Hamiltonian is explicitly given as

$$
\begin{aligned}
& H_{\sigma}: \ell^{2}(\Gamma) \rightarrow \ell^{2}(\Gamma) \\
& H_{\sigma}=\sum_{\gamma \in S} U(\gamma)
\end{aligned}
$$

and is clearly by definition a bounded self adjoint operator. Notice that the Hamiltonian can be decomposed as a sum of a free Hamiltonian containing the torsionfree generators and a term simulating Coulomb interactions, that contains the torsion generators.

$$
H_{\sigma}=H_{\sigma}^{\text {free }}+H_{\sigma}^{\text {interaction }}
$$

where

$$
H_{\sigma}^{\text {free }}=\sum_{j=0}^{g} U\left(A_{j}\right)+U\left(B_{j}\right)+\left(U\left(A_{j}\right)+U\left(B_{j}\right)\right)^{*}
$$

and

$$
H_{\sigma}^{\text {interaction }}=\sum_{i=1}^{n} U\left(C_{i}\right)+U\left(C_{i}\right)^{*} .
$$

Let $V \in \mathbb{C}(\Gamma, \sigma)$ be any "potential", and

$$
H_{\sigma, V}=H_{\sigma}+V
$$

Lemma 4.4. If $E \notin \operatorname{spec}\left(H_{\sigma, V}\right)$, then $P_{E} \in \mathcal{R}(\Gamma, \sigma)$, where $P_{E}=\chi_{[0, E]}\left(H_{\sigma, V}\right)$ is the spectral projection of the Hamiltonian to energy levels less than or equal to $E$.

Proof. Since $E \notin \operatorname{spec}\left(H_{\sigma, V}\right)$, then $P_{E}=\chi_{[0, E]}\left(H_{\sigma, V}\right)=\varphi\left(H_{\sigma, V}\right)$ for some smooth, compactly supported function $\varphi$. Now by definition, $H_{\sigma} \in \mathbb{C}(\Gamma, \sigma) \subset \mathcal{R}(\Gamma, \sigma)$, and since $\mathcal{R}(\Gamma, \sigma)$ is closed under the smooth functional calculus by the result of [Ji]], it follows that $P_{E} \in \mathcal{R}(\Gamma, \sigma)$.

Therefore by Corollary 4.3 and the discussion following it, we have,

Theorem 4.5 (Fractional Quantum Hall Effect). Suppose that the Fermi energy level E lies in a gap of the spectrum of the Hamiltonian $H_{\sigma, V}$, then the Hall conductance

$$
\sigma_{E}=\operatorname{tr}^{K}\left(P_{E}, P_{E}, P_{E}\right)=\operatorname{tr}_{c}\left(P_{E}, P_{E}, P_{E}\right) \in \phi \mathbb{Z}
$$

That is, the Hall conductance has plateaux which are integer multiples of $\phi$ on any gap in the spectrum of the Hamiltonian, where $-\phi=2(1-g)+(\nu-n) \in \mathbb{Q}$ is the orbifold Euler characteristic of $\Sigma\left(g ; \nu_{1}, \ldots, \nu_{n}\right)$.

Remarks 4.6. The set of possible denominators $\phi$ for low genus coverings can be derived easily from the results of Bro and is reproduced in the second table in the next section. It is plausible that this Theorem might shed light on the mathematical mechanism responsible for fractional quantum numbers that occur in the Quantum Hall Effect, as we attempt to explain in the following section. 


\section{Fractional Quantum numbers: Phenomenology}

We first discuss the characteristics of our model explaining the appearance of fractional quantum numbers in the quantum Hall effect. In particular, we point out the main advantages and limitations of the model.

Our model is a single electron model. It is well known that the FQHE is a consequence of the Coulomb interaction between electrons, hence it should not be seen by a single particle model. However, in our setting, the negative curvature of the hyperbolic structure provides a geometric replacement for interaction. The equivalence between negative curvature and interaction is well known from the case of classical mechanics where the Jacobi equation for a single particle moving on a negatively curved manifold can be interpreted as the Newton equation for a particle moving in the presence of a negative potential energy Arn.

The main advantage of this setting is that the fractions derived in this way are topological. In fact, they are obtained from an equivariant index theorem. Moreover, they are completely determined by the geometry of the orbifold. In fact, we have

$$
\phi=-\chi_{o r b}\left(\Sigma\left(g ; \nu_{1}, \ldots, \nu_{n}\right)\right) .
$$

Let us recall that the orbifold Euler characteristic $\chi_{\text {orb }}(\Sigma)$ of an orbifold $\Sigma$, is a rational valued invariant that is completely specified by the following properties, cf. [Tan]:

1. it is multiplicative under orbifold covers;

2. it coincides with the topological Euler characteristic in the case of a smooth surface;

3. it satisfies the volume formula,

$$
\chi_{\text {orb }}\left(\Sigma_{1} \cup \cdots \cup \Sigma_{k}\right)=\sum_{j=1}^{k} \chi_{o r b}\left(\Sigma_{j}\right)-\sum_{i, j} \chi_{o r b}\left(\Sigma_{i} \cap \Sigma_{j}\right)+\cdots(-1)^{k+1} \chi_{o r b}\left(\Sigma_{1} \cap \cdots \cap \Sigma_{k}\right),
$$

whenever all the intersections on the right hand side are suborbifolds of $\quad \Sigma_{1} \cup \cdots \cup \Sigma_{k}$, and all the $\Sigma_{j}$ are orbifolds of the same dimension.

This characterization allows for ease of computation and prediction of expected fractions.

Most notably, as pointed out in Bel+E+S, the topological nature of the Hall conductance makes it stable under small deformations of the Hamiltonian. Thus, this model can be easily generalized to systems with disorder, cf. [CHM]. This is a necessary step in order to establish the presence of plateaux $[\mathrm{Bel}+\mathrm{E}+\mathrm{S}]$.

The identifications of fractions with integer multiples of the orbifold Euler characteristic imposes some restrictions on the range of possible fractions from the geometry of the orbifolds. For instance, it is known from the Hurwitz theorem that the maximal order of a finite group acting by isometries on a smooth Riemann surface $\Sigma_{g^{\prime}}$ is $\#(G)=84\left(g^{\prime}-1\right)$. Moreover, this maximal order is always attained. Thus, the smallest possible fraction that appears in our model is $\phi=$ $\frac{2\left(g^{\prime}-1\right)}{84\left(g^{\prime}-1\right)}=1 / 42$. 
This is, in some respects, an advantage of the model, in as it gives very clear prediction on which fractions can occur, and at the same time its main limitation, in as we do not get a complete agreement between the set of fractions we obtain and the fractions that are actually observed in experiments on the FQHE.

In order to compare our predictions with experimental data, we restrict our attention to orbifolds with a torus or a sphere as underlying topological surface. Recall that, as explained above, we think of the hyperbolic structure induced by the presence of cone points on these surfaces as a geometric way of introducting interaction in this single electron model, hence we would consider equivalently the underlying surface with many interacting electrons (fractions observed in FQHE experiments) or as a hyperbolic surface with one electron.

We report a table of comparison between the values obtained experimentally and our prediction. Notice how the fraction 5/2 which appears in the experimental values and caused major problems of interpretation in the many-particle models appears here naturally as the orbifold Euler characteristic of $\Sigma(1 ; 6,6,6)$ (which we may as well refer to as the Devil's orbifold).

\begin{tabular}{|c||c|}
\hline experimental & $g=1$ or $g=0$ \\
\hline \hline $5 / 3$ & $\Sigma(1 ; 6,6)$ \\
\hline $4 / 3$ & $\Sigma(1 ; 3,3)$ \\
\hline $7 / 5$ & $\Sigma(0 ; 5,5,10,10)$ \\
\hline $4 / 5$ & $\Sigma(1 ; 5)$ \\
\hline $5 / 7$ & $\Sigma(0 ; 7,14,14)$ \\
\hline $2 / 3$ & $\Sigma(1 ; 3)$ \\
\hline $3 / 5$ & $\Sigma(0 ; 5,10,10)$ \\
\hline $4 / 7$ & $\Sigma(0 ; 7,7,7)$ \\
\hline $5 / 9$ & $? ? ?$ \\
\hline $4 / 9$ & $\Sigma(0 ; 3,9,9)$ \\
\hline $3 / 7$ & $? ? ?$ \\
\hline $2 / 5$ & $\Sigma(0 ; 5,5,5)$ \\
\hline $1 / 3$ & $\Sigma(0 ; 3,6,6)$ \\
\hline $5 / 2$ & $\Sigma(1 ; 6,6,6)$ \\
\hline
\end{tabular}

Despite the small number of discrepancies in the table above, the agreement between values of orbifold Euler characteristics and experimentally observed fractions in the quantum Hall effect is far from being satisfactory. In particular, not only there is a small number of observed values which are not orbifold Euler characteristics, but there are also many rational numbers that are realized as orbifold Euler characteristics, which do not seem to appear among the experimental data. For instance, by looking at the values of the next table, reported also in figure 1 , we see clearly that we have some fractions with even denominator, such as $1 / 4,1 / 2$, and $1 / 6$, which do not correspond to experimental values. As pointed out in the introduction, the reason for this discrepancy is that a more sophisticated model for the Coulomb interaction is needed in general.

In the remaining of this section, we discuss some phenomenology, with particular emphasis on the nature of the cone points and the role of the minimal genus of the covering surface $\Sigma_{g^{\prime}}$. We hope to return to these topics in some future work. 
Every orbifold $\Sigma\left(g ; \nu_{1}, \ldots, \nu_{n}\right)$ is obtained as a quotient of a surface $\Sigma_{g^{\prime}}$ with respect to the action of a finite group $G$, cf. [S]]. In general both $g^{\prime}$ and $G$ are not unique. For instance, the orbifold $\Sigma(1 ; 2,2)$ is obtained as the quotient of $\Sigma_{2}$ by the action of $\mathbb{Z}_{2}$, or as the quotient of $\Sigma_{3}$ by the action of $\mathbb{Z}_{4}$, or by the action of $\mathbb{Z}_{2} \times \mathbb{Z}_{2}$, cf. Bro. For every $\Sigma\left(g ; \nu_{1}, \ldots, \nu_{n}\right)$ there is a minimal $g^{\prime}$ such that the orbifold is obtained as a quotient of $\Sigma_{g^{\prime}}$ by a finite group action. In [Bro], Broughton has derived a complete list of all the good two dimensional orbifolds which are quotients of Riemann surfaces $\Sigma_{g^{\prime}}$ with genus $g^{\prime}=2$ or 3 .

In a physical model one can distinguish between two types of disorder: a mobility disorder and a sample disorder, cf. Bel+E+S. We can argue phenomenologically that, if an orbifold can be realized by a covering of low genus, this corresponds to a lower density of atoms in the sample, as opposed to the case of a surface of high genus, as one can see by looking at the Cayley graph of $\Gamma_{g^{\prime}}$. Thus, we can consider the minimal genus of the smooth coverings as a measure of mobility. This means that, in an experiment, the fractions derived from orbifolds with low genus coverings will be easier to observe (have more clearly marked plateaux) than fractions which are only realized by quotients of surfaces of higher genus.

Thus, we can consider the list of examples given in Bro and compute the corresponding fractions. We list the result in the following table.

\begin{tabular}{|c|c||c|}
\hline$\phi$ & $g^{\prime}=2$ & $g^{\prime}=3$ \\
\hline \hline $4 / 3$ & & $\Sigma(0 ; 3,3,3,3,3) \Sigma(1 ; 3,3)$ \\
\hline $2 / 3$ & $\Sigma(0 ; 3,3,3,3)$ & $\Sigma(0 ; 2,2,6,6) \Sigma(0 ; 2,3,3,6) \Sigma(0 ; 2,2,2,2,3) \Sigma(1 ; 3)$ \\
\hline $4 / 7$ & & $\Sigma(0 ; 7,7,7)$ \\
\hline $1 / 2$ & $\Sigma(0 ; 2,2,4,4)^{*} \Sigma(0 ; 2,2,2,2,2)^{*}$ & $\Sigma(0 ; 4,8,8) \Sigma(1 ; 2)$ \\
\hline $4 / 9$ & & $\Sigma(0 ; 3,9,9)$ \\
\hline $2 / 5$ & $\Sigma(0 ; 5,5,5)$ & $\Sigma(0 ; 2,12,12) \Sigma(0 ; 3,4,12) \Sigma(0 ; 4,4,6) \Sigma(0 ; 2,2,2,6)$ \\
\hline $1 / 3$ & $\Sigma(0 ; 3,6,6) \Sigma(0 ; 2,2,3,3)^{*}$ & \\
\hline $1 / 4$ & $\Sigma(0 ; 2,8,8)^{*} \Sigma(0 ; 4,4,4)^{*} \Sigma(0 ; 2,2,2,4)^{*}$ & $\Sigma(0 ; 3,7,7)$ \\
\hline $1 / 5$ & $\Sigma(0 ; 2,5,10)$ & $\Sigma(0 ; 2,4,12) \Sigma(0 ; 3,3,6)$ \\
\hline $4 / 21$ & & \\
\hline $1 / 6$ & $\Sigma(0 ; 3,4,4)^{*} \Sigma(0 ; 2,6,6)^{*} \Sigma(0 ; 2,2,2,3)^{*}$ & \\
\hline $1 / 8$ & $\Sigma(0 ; 2,4,8)^{*}$ & $\Sigma(0 ; 2,3,7)$ \\
\hline $1 / 12$ & $\Sigma(0 ; 2,4,6)^{*} \Sigma(0 ; 3,3,4)^{*}$ & \\
\hline $1 / 24$ & $\Sigma(0 ; 2,3,8)^{*}$ & \\
\hline $1 / 42$ & & \\
\hline
\end{tabular}

In the table the orbifolds that are markes with a $*$ can be realized both as quotient of $\Sigma_{2}$ and of $\Sigma_{3}$. It seems also reasonable to think that if the same fraction is realized by several different orbifolds, for fixed $g^{\prime}$, then the corresponding plateau will be more clearly marked in the experiment. This would make $\phi=1 / 3$ the most clearly pronounced plateau, which is in agreement with the experimental data. However, higher genus corrections are not always negligible. In fact, by only considering genus $g^{\prime}=2$ and $g^{\prime}=3$ contributions, we would expect a more marked plateau for the fraction $\phi=2 / 3$ than for the fraction $\phi=2 / 5$, and the experimental results show that this is not the case. It seems important to observe that this model produces equally easily examples of fractions with odd or even denominators (e.g. $\phi=1 / 4$ appears in the table above). It is interesting to compare this datum with the difficulty encountered within other models in 
explaining the appearance of the fraction $5 / 2$ in the experiments. Its presence is only justified by introducing a different physical model (the so called non-abelian statistics). In figure 1 w we sketch the plateaux as they would appear in the result of an experiment, using only the low genus $g^{\prime}=2$ and $g^{\prime}=3$ approximation.

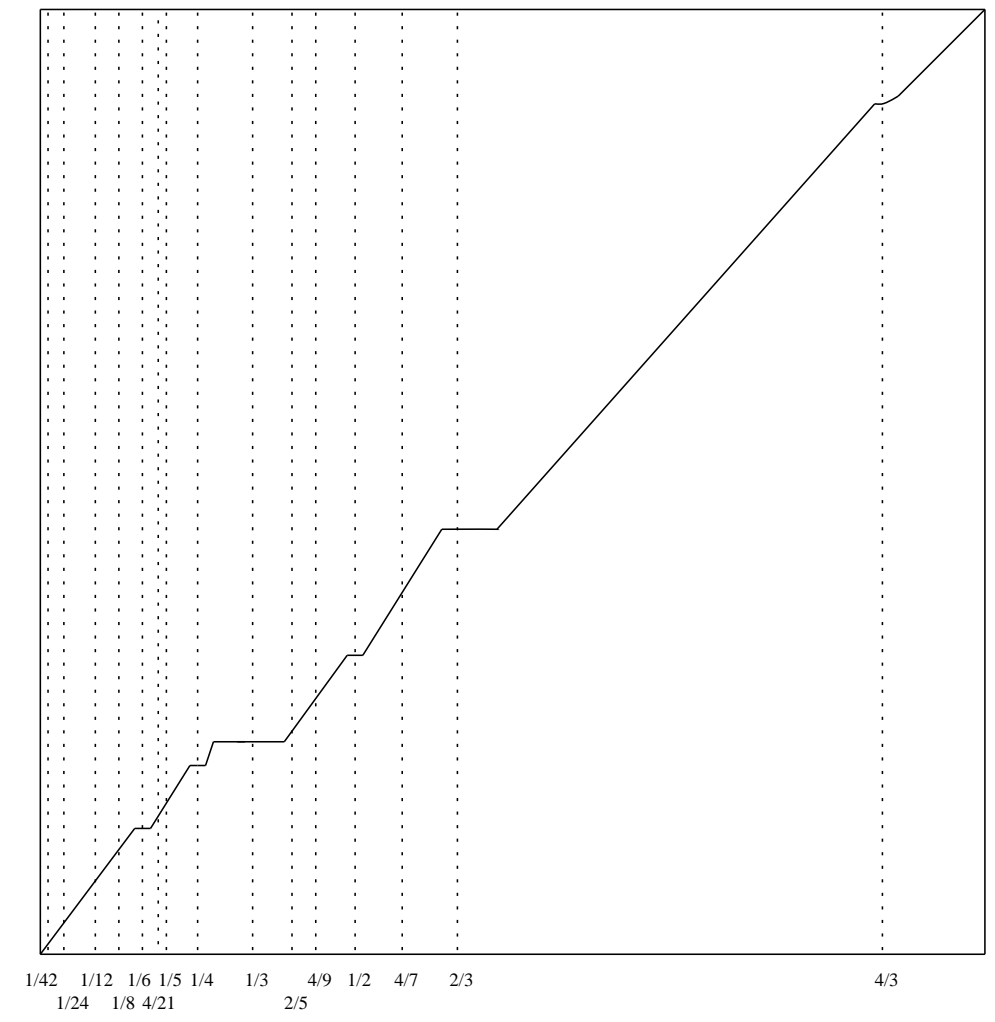

FiguRE 1. Phenomenology of fractions in the low genus approximation

As we already mentioned in the introduction, both the hyperbolic structure and the cone points are essential in order to have fractional quantum numbers. In fact, $\phi$ is an integer whenever the hyperbolic orbifold is smooth, i.e. whenever $1=\nu_{1}=\ldots=\nu_{n}$, which is the case considered in CHMM. On the other hand, by direct inspection, it is possible to see that all euclidean orbifolds also produce only integer values of $\phi$. (Notice that sometimes hyperbolic orbifolds with cone points may still produce integers: the orbifold $\Sigma(1 ; 2,2)$ has $\phi=1$, cf. [Bro].) Models of FQHE on euclidean orbifolds have been considered, in a different, string-theoretic context, e.g. SSk-Th].

We can argue that the cone points can also be thought of as a form of "disorder". In fact, we may identify the preimage of the cone points in the universal covering $\mathbb{H}$ with sample disorder (with respect to the points in the Cayley graph of $\Gamma_{g^{\prime}}$ ). The same fraction can often be obtained by orbifolds with a varying number of cone points (for fixed $g^{\prime}$ ), as illustrated in the previous table. This can be rephrased by saying that the system allows for more or less sample disorder, and in some cases this can be achieved without affecting the mobility measured by $g^{\prime}$. 


\section{APPENDIX}

The main purpose of this appendix is to establish Lemma F, which is used in the paper. We follow closely the approach in $\mathrm{BrSu}$. We use the notation of the previous sections. Let $A$ be an operator on $L^{2}(\widetilde{M}, \widetilde{S} \otimes E)$ with Schwartz kernel $k_{A}$ and also commuting with the given $(\Gamma, \bar{\sigma})$-action. Then one has

$$
e^{i \phi_{\gamma}(x)} k_{A}(\gamma x, \gamma y) e^{-i \phi_{\gamma}(y)}=k_{A}(x, y) \quad \forall \gamma \in \Gamma,
$$

where we have identified the fibre at $x \in \widetilde{M}$ with the fibre at $\gamma x \in \widetilde{M}$. If $k_{A}$ is smooth, then one can define the von Neumann trace just as Atiyah did in the untwisted case,

$$
\operatorname{tr}\left(k_{A}\right)=\int_{\mathcal{F}} \underline{\operatorname{tr}}\left(k_{A}(x, x)\right) d x
$$

where $\mathcal{F}$ denotes a fundamental domain for the action of $\Gamma$ on $\widetilde{M}$ and where $\underline{\operatorname{tr}}$ denotes the pointwise or local trace. The von Neumann trace is well defined, since as a consequence of (10), $\underline{\operatorname{tr}}\left(k_{A}(x, x)\right)$ is a $\Gamma$-invariant function on $\widetilde{M}$. The following lemma establishes that it is a trace.

Lemma (A). Let $A, B$ be operators on $L^{2}(\widetilde{M}, \widetilde{S} \otimes E)$ with smooth Schwartz kernels and also commuting with the given $(\Gamma, \bar{\sigma})$-action. Then one has

$$
\operatorname{tr}(A B)=\operatorname{tr}(B A) .
$$

Proof. Let $k_{A}, k_{B}$ denote the smooth Schwartz kernels of $A, B$ respectively, and $k_{A B}, k_{B A}$ denote the smooth Schwartz kernels of $A B, B A$ respectively. Then one has

$$
\begin{aligned}
\operatorname{tr}(A B-B A) & =\int_{x \in \mathcal{F}} \underline{\operatorname{tr}}\left(k_{A B}(x, y)-k_{B A}(x, y)\right) \\
& =\int_{x \in \mathcal{F}} \int_{y \in \widetilde{M}} \underline{\operatorname{tr}}\left(k_{A}(x, y) k_{B}(y, x)-k_{B}(x, y) k_{A}(y, x)\right) \\
& =\sum_{\gamma \in \Gamma} \int_{x \in \mathcal{F}} \int_{y \in \mathcal{F}} \underline{\operatorname{tr}}\left(k_{A}(x, \gamma y) k_{B}(\gamma y, x)-k_{B}(x, \gamma y) k_{A}(\gamma y, x)\right) \\
& =0
\end{aligned}
$$

since each term in the summand vanishes by symmetry, and we have used the fact that the fundamental domain $\mathcal{F}$ is compact in order to interchange the order of the summation and integral.

We will also adopt a more operator theoretic approach. Let $\mathcal{H}=L^{2}\left(\mathcal{F},\left.\widetilde{S} \otimes E\right|_{\mathcal{F}}\right)$. Then $\Phi$ : $L^{2}(\widetilde{M}, \widetilde{S} \otimes E) \cong \ell^{2}(\Gamma, \mathcal{H})$ is given by $(\Phi s)(\gamma)=R_{\mathcal{F}}\left(T_{\gamma} s\right) \forall \gamma \in \Gamma$, where $R_{\mathcal{F}}: L^{2}(\widetilde{M}, \widetilde{S} \otimes E) \rightarrow \mathcal{H}$ denotes the restriction map to the fundamental domain $\mathcal{F}$.

As in section 1 , let $W^{*}(\sigma)$ denote the commutant, i.e.

$$
W^{*}(\sigma)=\left\{A \in \ell^{2}(\Gamma, \mathcal{H}):\left[T_{\gamma}, A\right]=0 \quad \forall \gamma \in \Gamma\right\}
$$

Then one has the following simple lemma,

Lemma $(\mathrm{B}) . W^{*}(\sigma)$ is a semifinite von Neumann algebra. 
Proof. We need to show that $W^{*}(\sigma)$ is a $*$-algebra which is weakly closed. We will establish that it is has a semifinite trace a bit later on.

Let $A, B \in W^{*}(\sigma)$. Since $\left[T_{\gamma}, A B\right]=\left[T_{\gamma}, A\right] B+A\left[T_{\gamma}, B\right]$, it follows that $A B \in W^{*}(\sigma)$. Since $\left[T_{\gamma}, A\right]=-\left[T_{\gamma}^{*}, A^{*}\right]=-\left[T_{\gamma^{-1}}, A^{*}\right]$ it follows $A^{*} \in W^{*}(\sigma)$. Clearly the identity operator is in $W^{*}(\sigma)$. Finally, if $A_{n} \in W^{*}(\sigma) \forall n \in \mathbb{N}$ and $A_{n}$ converges weakly to $A$, it follows that for all $\gamma \in \Gamma, T_{\gamma} A_{n}$ converges weakly to $T_{\gamma} A$ and also to $A T_{\gamma}$. By uniqueness of weak limits, we deduce that $A \in W^{*}(\sigma)$.

For $A \in W^{*}(\sigma)$, define its generalized Fourier coefficients $\widehat{A}(\gamma) \in B(\mathcal{H})$ as

$$
\widehat{A}(\gamma) v=T_{\gamma}\left(A \delta_{1}^{v}\right)(1)
$$

where $\delta_{1}^{v} \in \ell^{2}(\Gamma, \mathcal{H})$ is defined for all $v \in \mathcal{H}$ as

$$
\delta_{1}^{v}(\gamma)=\left\{\begin{array}{l}
v \text { if } \gamma=1 \\
0 \text { otherwise }
\end{array}\right.
$$

Since $T_{\gamma} \delta_{1}^{v}\left(\gamma^{\prime}\right)=\delta_{1}^{v}\left(\gamma^{\prime} \gamma\right) \sigma\left(\gamma^{\prime}, \gamma\right)$, one has

$$
T_{\gamma} \delta_{1}^{v}\left(\gamma^{\prime}\right)=\left\{\begin{array}{l}
v \text { if } \gamma^{\prime}=\gamma^{-1} \\
0 \text { otherwise }
\end{array}\right.
$$

since $\sigma\left(\gamma^{-1}, \gamma\right)=1 \forall \gamma \in \Gamma$. In particular, it follows that for all $f \in \ell^{2}(\Gamma, \mathcal{H})$, one has

$$
f(\gamma)=\sum_{\gamma_{1} \gamma_{2}=\gamma} T_{\gamma_{1}} \delta_{1}^{f\left(\gamma_{2}\right)}
$$

so that one has the following Fourier expansion

$$
\begin{aligned}
A f(\gamma) & =\sum_{\gamma_{1} \gamma_{2}=\gamma} A T_{\gamma_{1}} \delta_{1}^{f\left(\gamma_{2}\right)}=\sum_{\gamma_{1} \gamma_{2}=\gamma} T_{\gamma_{1}} A \delta_{1}^{f\left(\gamma_{2}\right)} \\
& =\sum_{\gamma_{1} \gamma_{2}=\gamma} \widehat{A}\left(\gamma_{1}\right)\left(f\left(\gamma_{2}\right)\right) .
\end{aligned}
$$

The following elementary properties are satisfied by the Fourier coefficients.

Lemma (C). For $A, B \in W^{*}(\sigma)$ and for all $\gamma \in \Gamma$, for all $f \in \ell^{2}(\Gamma, \mathcal{H})$, one has

(1) $A f(\gamma)=\sum_{\gamma_{1} \gamma_{2}=\gamma} \widehat{A}\left(\gamma_{1}\right)\left(f\left(\gamma_{2}\right)\right)$;

(2) $\widehat{A}^{*}(\gamma)=\left(\widehat{A}\left(\gamma^{-1}\right)\right)^{*}$;

(3) $\widehat{A B}(\gamma)=\sum_{\gamma_{1} \gamma_{2}=\gamma} \widehat{A}\left(\gamma_{1}\right) \widehat{B}\left(\gamma_{2}\right)$;

(4) $\widehat{A A^{*}}(1)=\sum_{\gamma} \widehat{A}(\gamma) \widehat{A}(\gamma)$;

(5) $\|A\| \leq \sum_{\gamma}\|\widehat{A}(\gamma)\|$;

(6) $\widehat{A-B}(\gamma)=\widehat{A}(\gamma)-\widehat{B}(\gamma)$. 
Proof. The proof follows by straightforward calculations as done above. The reader is warned that the righthand side of the inequality in part (5) is not necessarily finite.

Define $C_{o}(\Gamma, \mathcal{K})$ to be the set of all $A \in W^{*}(\sigma)$ such that $\widehat{A}(\gamma) \in \mathcal{K} \forall \gamma \in \Gamma$, and $\widehat{A}(\gamma)=0$ for all but finitely many $\gamma \in \Gamma$. Then the completion of $C_{o}(\Gamma, \mathcal{K})$ with respect to the operator norm is denoted, as in section 1 of [MM], by $C_{r}^{*}(\Gamma, \sigma) \otimes \mathcal{K}$, and called the twisted crossed product algebra associated to the twisted action $(\alpha, \sigma)$. Then one has the following useful containment criterion,

Lemma (D). If $A \in W^{*}(\sigma)$ and also satisfies $\sum_{\gamma}\|\widehat{A}(\gamma)\|<\infty$, then $A \in C_{r}^{*}(\Gamma, \sigma) \otimes \mathcal{K}$.

If $A \in W^{*}(\sigma)$ and also satisfies $\sum_{\gamma} d(\gamma, 1)^{k}\|\widehat{A}(\gamma)\|<\infty$, for all positive integers $k$, then $A \in \mathcal{R}(\Gamma, \sigma)$.

Proof. Let $K_{1} \subset K_{2} \subset \cdots$ be a sequence of finite subsets of $\Gamma$ which is an exhaustion of $\Gamma$, i.e. $\bigcup_{j \geq 1} K_{j}=\Gamma$. For all $j \in \mathbb{N}$, define $A_{j} \in W^{*}(\sigma)$ by

$$
\widehat{A_{j}}(\gamma)=\left\{\begin{array}{l}
\widehat{A}(\gamma) \text { if } \gamma \in K_{j} \\
0 \text { otherwise }
\end{array}\right.
$$

Then in fact $A_{j} \in C_{o}(\Gamma, \mathcal{K})$ by definition, and using the previous lemma, we have

$$
\begin{aligned}
\left\|A-A_{j}\right\| & \leq \sum_{\gamma}\left\|\widehat{A-A_{j}}(\gamma)\right\| \\
& =\sum_{\gamma}\left\|\widehat{A}(\gamma)-\widehat{A_{j}}(\gamma)\right\| \\
& =\sum_{\gamma \in \Gamma \backslash K_{j}}\|\widehat{A}(\gamma)\| .
\end{aligned}
$$

By hypothesis, $\sum_{\gamma}\|\widehat{A}(\gamma)\|<\infty$, therefore $\sum_{\gamma \in \Gamma \backslash K_{j}}\|\widehat{A}(\gamma)\| \rightarrow 0$ as $j \rightarrow \infty$, since $K_{j}$ is an increasing exhaustion of $\Gamma$. This proves that $A \in C_{r}^{*}(\Gamma, \sigma) \otimes \mathcal{K}$.

The second part is clear from the definition, once we identify $\mathcal{R}$ with the algebra of sequences

$$
\left\{\left(a_{\gamma}\right)_{\gamma \in \Gamma}\left|\sup _{\gamma \in \Gamma} d(\gamma, 1)^{k}\right| a_{\gamma} \mid<\infty \forall k \in \mathbb{N}\right\} .
$$

The following off-diagonal estimate is well known, cf. BrSu.

Lemma (E). Let $D=\widetilde{\not D}_{E}^{+} \otimes \nabla^{s}$ be a twisted Dirac operator. Then the Schwartz kernel $k(t, x, y)$ of the heat operator $e^{-t D^{*} D}$ is smooth $\forall t>0$. It also satisfies the following off-diagonal estimate

$$
|k(t, x, y)| \leq C_{1} t^{-n / 2} e^{-C_{2} d(x, y)^{2} / t}
$$


uniformly in $(0, T] \times \widetilde{M} \times \widetilde{M}$ for any $T>0$, where $d$ denotes the Riemannian distance function on $\widetilde{M}$. The same result is true for the the Schwartz kernel of the heat operator $e^{-t D D^{*}}$.

Lemma (F). Let $D=\widetilde{\partial}_{E}^{+} \otimes \nabla^{s}$ be a twisted Dirac operator. Then $e^{-t D^{*} D}, e^{-t D D^{*}} \in \mathcal{R}(\Gamma, \sigma) \subset$ $C_{r}^{*}(\Gamma, \sigma) \otimes \mathcal{K} \quad \forall t>0$.

Proof. By the Lemma above, it follows that $e^{-t D^{*} D}, e^{-t D D^{*}}$ are bounded operators commuting with the given twisted action, i.e. $e^{-t D^{*} D}, e^{-t D D^{*}} \in W^{*}(\Gamma, \mathcal{H})$. Since the Schwartz kernels of $\widehat{e^{-t D^{*} D}}(\gamma), \widehat{e^{-t D D^{*}}}(\gamma)$ are smooth $\forall \gamma \in \Gamma$ by the Lemma above, it follows that $\widehat{e^{-t D^{*} D}}(\gamma), \widehat{e^{-t D D^{*}}}(\gamma) \in$ $\mathcal{K} \forall \gamma \in \Gamma$. Let $d_{\Gamma}$ denote the word metric with respect to a given finite set of generators, and $d$ the Riemannian metric on $\widetilde{M}$. Then it is well known that

$$
d_{\Gamma}\left(\gamma_{1}, \gamma_{2}\right) \leq C_{3}\left(\inf _{x, y \in \widetilde{M}} d\left(\gamma_{1} x, \gamma_{2} y\right)+1\right)
$$

for some positive constant $C_{3}$. By the Lemma 5 above, one has,

$$
\left\|\widehat{e^{-t D^{*} D}}(\gamma)\right\| \leq C_{4} e^{-C_{5} d_{\Gamma}(\gamma, 1)^{2}}
$$

for some positive constants $C_{4}, C_{5}$, and a similar estimate holds for $\widehat{e^{-t D D^{*}}}(\gamma)$. Setting $r(\gamma)=$ $d_{\Gamma}(\gamma, 1)$ observe that one has the estimate

$$
\#\{\gamma \in \Gamma \mid r(\gamma) \leq R\} \leq C_{6} e^{C_{7} R}
$$

for some positive constants $C_{6}, C_{7}$, since the volume growth rate of $\Gamma$ is at most exponential. Therefore one has

$$
\sum_{\gamma} d(\gamma, 1)^{k}\left\|\widehat{e^{-t D^{*} D}}(\gamma)\right\|<\infty \quad \text { and } \quad \sum_{\gamma} d(\gamma, 1)^{k}\left\|\widehat{e^{-t D D^{8}}}(\gamma)\right\|<\infty
$$

for all positive integers $k$. By the Lemma above, it follows that $e^{-t D^{*} D}, e^{-t D D^{*}} \in \mathcal{R}(\Gamma, \sigma) \subset$ $C_{r}^{*}(\Gamma, \sigma) \otimes \mathcal{K} \forall t>0$.

\section{REFERENCES}

[Arn] V.I. Arnold, Mathematical methods of classical mechanics, Graduate Texts in Mathematics, Vol.60, Springer 1978.

[At] M.F. Atiyah, Elliptic operators, discrete groups and Von Neumann algebras, Astérisque 32-33 (1976), 43-72.

$[\mathrm{Av}+\mathrm{S}+\mathrm{Y}] \quad$ J. Avron, R. Seiler, I. Yaffe, Adiabatic theorems and applications to the integer quantum Hall effect, Commun. Math. Phys. 110 (1987), 33-49.

[BC] P. Baum and A. Connes, Chern Character for discrete groups, in A fete of Topology, Academic Press (1988) 163-232.

[BCH] P. Baum, A. Connes and N. Higson, Classifying space for proper actions and $K$-theory of group $C^{*}$-algebras, Contemp. Math. 167 (1994) 241-291.

$[$ Bel + E $+\mathrm{S}] \quad$ J. Bellissard, A. van Elst, H. Schulz-Baldes, The non-commutative geometry of the quantum Hall effect, J. Math. Phys. 35 (1994), 5373-5451.

[Bost] J. Bost, Principe d'Oka, $K$-théorie et systémes dynamiques non commutatifs, Invent. Math. 101 (1990), no. 2, 261-333. 
[Bro] A. Broughton, Classifying finite group actions on surfaces of low genus, J. Pure Appl. Algebra 69 (1991), no. 3, 233-270.

[BrSu] J. Brüning, T. Sunada, On the spectrum of gauge-periodic elliptic operators. Méthodes semiclassiques, Vol. 2 (Nantes, 1991). Astérisque 210 (1992), 65-74.

[CHMM] A. Carey, K. Hannabuss, V. Mathai and P. McCann, Quantum Hall Effect on the hyperbolic plane, Commun. Math. Physics, 190 no. 3 (1998) 629-673.

[CHM] A. Carey, K. Hannabuss, V. Mathai, Quantum Hall effect on the hyperbolic plane in the presence of disorder, Lett. Math. Phys. 47 (1999), no. 3, 215-236

[Co] A. Connes, Non commutative differential geometry, Publ. Math. I.H.E.S. 62 (1986), 257-360.

[Co2] A. Connes, Noncommutative geometry, Academic Press, Inc., San Diego, CA, (1994).

[CM] A. Connes, H. Moscovici, Cyclic cohomology, the Novikov conjecture and hyperbolic groups, Topology 29 (1990), 345-388.

[Far] C. Farsi, K-theoretical index theorems for good orbifolds, Proc. Amer. Math. Soc. 115 (1992) 769-773.

[Froh] J. Frohlich, Transport in thermal equilibrium, gapless modes, and anomalies, in Festschrift for the 40th anniversary of the IHES, Publ. Math. I.H.E.S. (1998) 81-97.

[FuSt] M. Furuta and B. Steer, Seifert fibred homology 3-spheres and the Yang-Mills equations on Riemann surfaces with marked points, Adv. Math. 96 (1992), no. 1, 38-102.

$[\mathrm{GH}]$

$[\mathrm{Gr}]$

$[\mathrm{Gr} 2]$

$[\mathrm{Ji}]$

P. Griffiths and J. Harris, Principles of algebraic geometry, Wiley, New York, 1978.

M. Gromov, Volume and bounded cohomology, Publ. Math. I.H.E.S. 56 (1982), 5-99.

M. Gromov, Kähler-hyperbolicity and $L^{2}$ Hodge theory, J. Diff. Geom. 33 (1991), 263-292.

R. Ji, Smooth dense subalgebras of reduced group $C^{*}$-algebras, Schwartz cohomology of groups and cyclic cohomology, Jour. Func. Anal. 107 (1992), 1-33.

[Kaw] T. Kawasaki, The index of elliptic operators over V-manifolds, Nagoya Math. Jour. 84 (1981) $135-157$.

[Kaw2] T. Kawasaki, The signature theorem for $V$-manifolds, Topology, 17 (1978), no. 1, 75-83.

[MM] M. Marcolli, V. Mathai, Twisted index theory on good orbifolds, I: noncommutative Bloch theory, Communications in Contemporary Mathematics 1 no. 4 (1999) 553-587.

[Ma1] V. Mathai, K-theory of twisted group $C^{*}$-algebras and positive scalar curvature, Rothenberg Festschrift, Contemp. Math. 231 (1999) 203-225.

[Mos] G. Mostow, Strong rigidity of symmetric spaces, Ann. Math. Studies, 78 (1973), Princeton University Press.

[Patt] S.J. Patterson, On the cohomology of Fuchsian groups, Glasgow Math. J. 16 (1975), no. 2, 123140.

[Sc] P. Scott, The geometries of 3-manifolds, Bull. Lond. Math. Soc. 15 (1983) 401-487.

[Si] I.M. Singer, Some remarks on operator theory and index theory, in $K$-theory and operator algebras, Lecture Notes in Math., Vol. 575, Springer, Berlin, (1977) 128-138.

[Sk-Th] S. Skoulakis, S. Thomas, Orbifold duality symmetries and quantum Hall systems, Nucl.Phys. B 538 (1999) 659-684.

[Sun] T. Sunada, A discrete analogue of periodic magnetic Schrödinger operators, Contemp. Math. 173 (1994), 283-299.

[Tan] C. Tanasi, The Euler-Poincaré characteristic of two-dimensional orbifolds, Rend. Sem. Mat. Univ. Politec. Torino 45 (1987) 133-155.

[Xia] J. Xia, Geometric invariants of the quantum Hall effect, Commun. Math. Phys. 119 (1988), 29-50.

Department of Mathematics, Massachussetts Institute of Technology, Cambridge, Mass., USA

E-mail address: matilde@math.mit.edu

Department of Mathematics, University of Adelaide, Adelaide 5005, Australia

E-mail address: vmathai@maths.adelaide.edu.au 